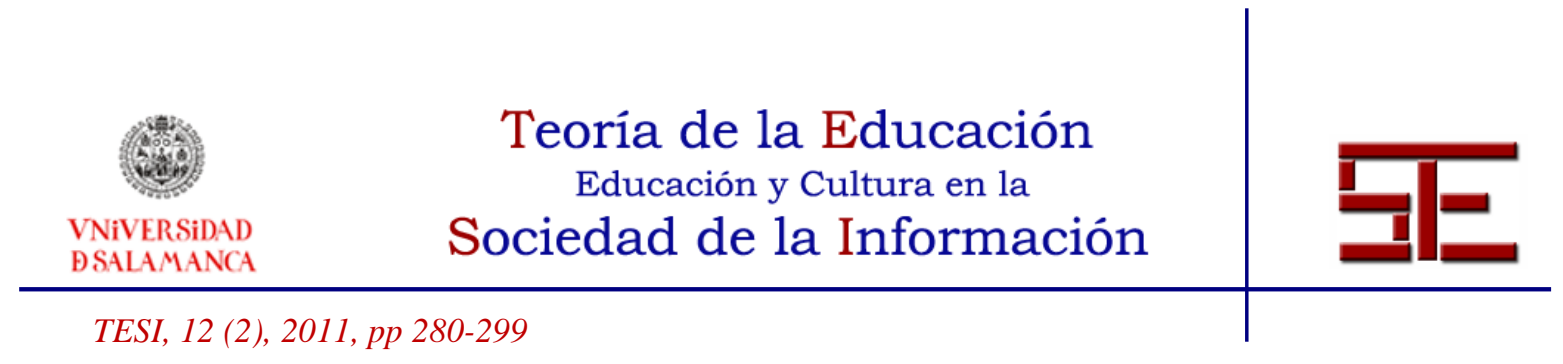

\title{
LAS HABILIDADES DE COMUNICACIÓN COMO ELEMENTO DE INNOVACIÓN EDUCATIVA: ESTRATEGIAS DE APRENDIZAJE
}

Resumen: Las estrategias de aprendizaje de la adquisición y recuperación de las habilidades de comunicación son un aspecto poco estudiado, por no decir que es un territorio todavía sin explorar. Conocer por parte de los alumnos de Magisterio, cómo se establecen esas estrategias y cómo se van incorporando al uso cotidiano esas habilidades de comunicación, se revela fundamental como elemento de innovación educativa.

El objetivo fundamental de esta investigación era analizar qué estrategias de aprendizaje se ponen en marcha cuando se trabaja la recuperación y adquisición de habilidades de en personas con problemas de comunicación.

La investigación se diseña como un estudio prospectivo y comparativo, con distribución aleatoria de los participantes en dos grupos (experimental y control).

Como primeras conclusiones, vemos que en los participantes del grupo experimental se ha incrementado la adquisición de estrategias de aprendizaje y ejecución de las mismas. Vemos que un programa en el que no sólo se entrene a la persona en ejercicios repetitivos de formulación de pregunta-respuesta, sino que además se incentive la búsqueda propia de soluciones ante problemas cotidianos, tiene grandes efectos beneficiosos, sobre todo en aspectos de adquisición de estrategias de aprendizaje y la adquisición de nuevos aprendizajes o recuerdo de anteriores aprendizajes, que el sujeto ya, poseía pero no demostraba o practicaba.

Palabras clave: Habilidades de comunicación; estrategias de aprendizaje; innovación; educación.

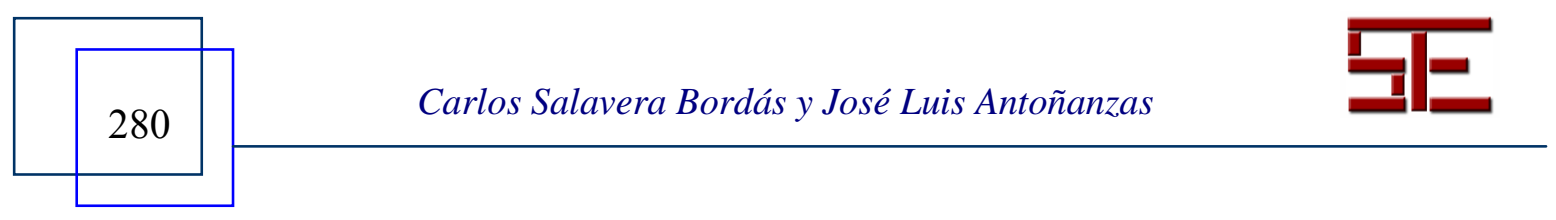




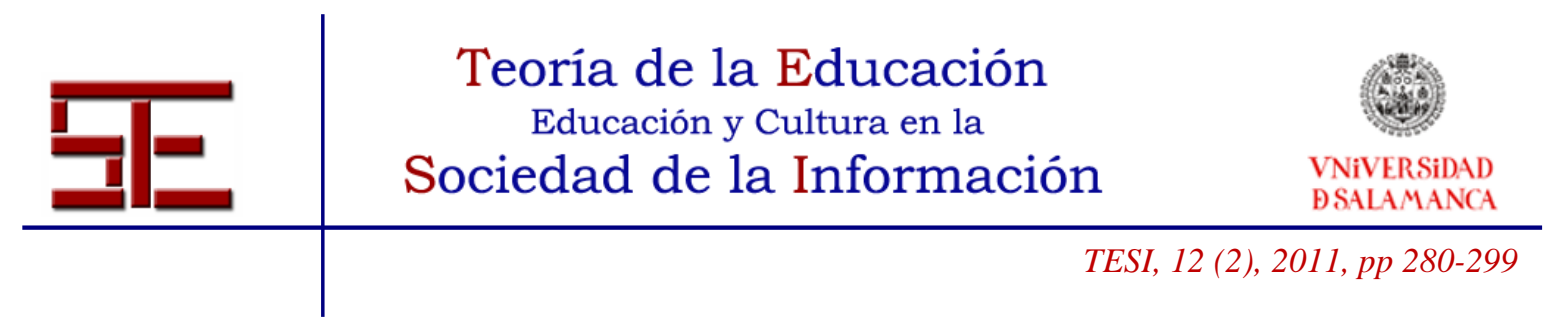

\title{
COMMUNICATION SKILLS FOR INNOVATION AS AN EDUCATIONAL STRATEGIES LEARNING
}

\begin{abstract}
Learning strategies of acquisition and retrieval skills. Communication is a poorly understood aspect, not to say that it is a territory unexplored. Known by students in teacher training, as establishing such strategies an how they are incorporating these everyday communication skills, be essential as an element of innovation education.
\end{abstract}

The main objective of this research was to examine what strategies learning are in place when working the recovery and acquisition of skills in people with communication problems.

The research was designed as a comparative prospective study with Random allocation of participants into two groups (experimental and control). As conclusions, we see that the experimental group participants increased acquisition of learning strategies and implementation of them. We see that a program that not only train the person making drills question-answer, but it also Search incentive own solutions to everyday problems has great beneficial effects, particularly in aspects of acquisition strategies learning and acquisition of new learning or memory of previous learning, the subject already possessed but not demonstrated or practice.

Keywords: Communication skills; learning strategies; innovation; education.

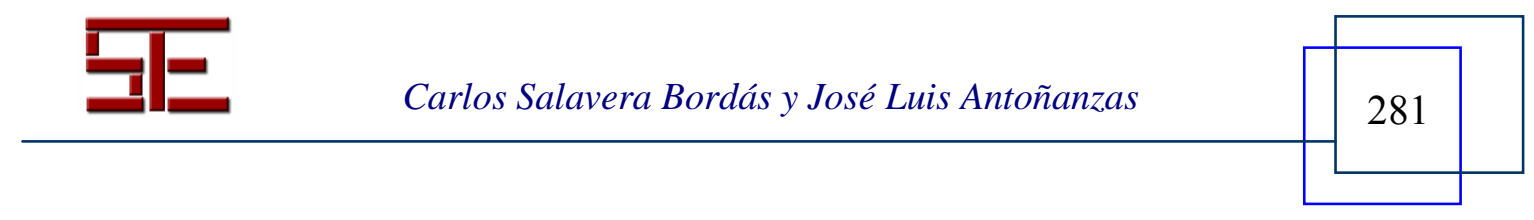




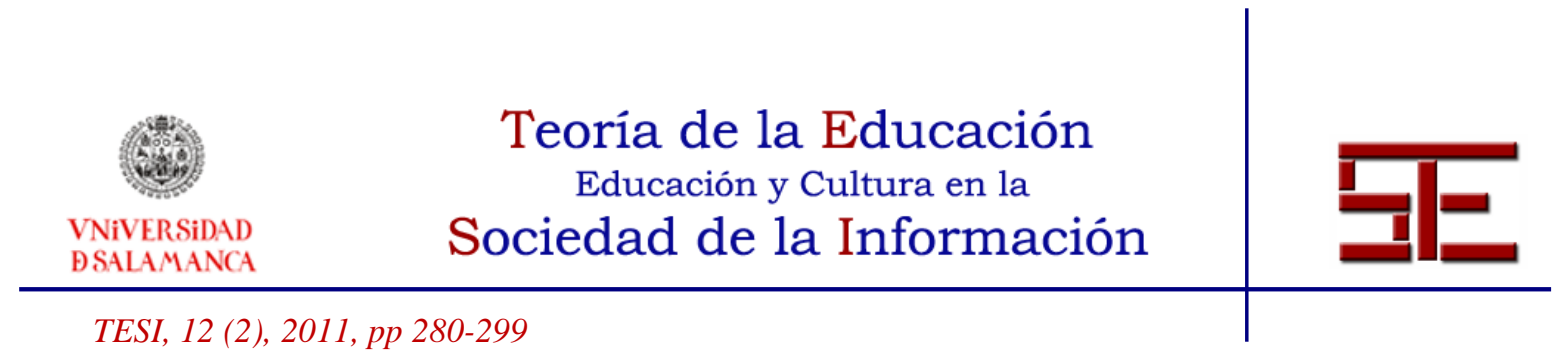

\section{LAS HABILIDADES DE COMUNICACIÓN COMO ELEMENTO DE INNOVACIÓN EDUCATIVA: ESTRATEGIAS DE APRENDIZAJE}

Fecha de recepción: 12/03/2011; fecha de aceptación: 14/05/2011; fecha de publicación: 27/07/2011

Carlos Salavera

salavera@unizar.es

Universidad de Zaragoza

José Luis Antoñanzas

jlantona@unizar.es

Universidad de Zaragoza

La comunicación es uno de los factores fundamentales a la hora de desarrollar la tarea docente. Las habilidades mostradas en este campo por los profesionales de la educación tienen un peso específico en los aprendizajes adquiridos y asimilados por sus alumnos. Trabajar las habilidades de comunicación se evidencia fundamental en la mejora y capacitación de las competencias profesionales (Caballo, 1993; Gil, 1984; Goldstein, Sprafkin; Gershaw \& Klein, 1989; Kelly, 1987; Nedrum, 1997). Las estrategias de aprendizaje de la adquisición y recuperación de las habilidades de comunicación son uno de los aspectos menos estudiados, por no decir que es un territorio todavía sin explorar. Conocer cómo se establecen esas estrategias y cómo se van incorporando al uso cotidiano esas habilidades de comunicación es el objetivo principal de este trabajo.

A la hora de analizar factores y variables que intervienen en la posible capacitación de los nuevos docentes, son una de las principales variables que influyen cuando se habla de desarrollo profesional (Andreu; Sanz y Serrat, 2009), marcando la evolución de su carrera docente.

En el trabajo día a día en el aula, una de las diferencias entre los aspirantes a futuros maestros, uno de los problemas que se observa, es la dificultad de algunos de ellos para mantener una conversación larga y extensa. Estas dificultades son en principio observables en grupos clínicos (Salavera, 1998, 1999; Salavera y Puyuelo, 2010), pero también en universitarios (Salavera; Tricás; Lucha; Gaspar; Herranz, y Jiménez, 1999).

Para ello se desarrollaron dos grupos. Por un lado, un grupo con un buen nivel de estructuración (un grupo que trabajase las habilidades de comunicación) y, por otro

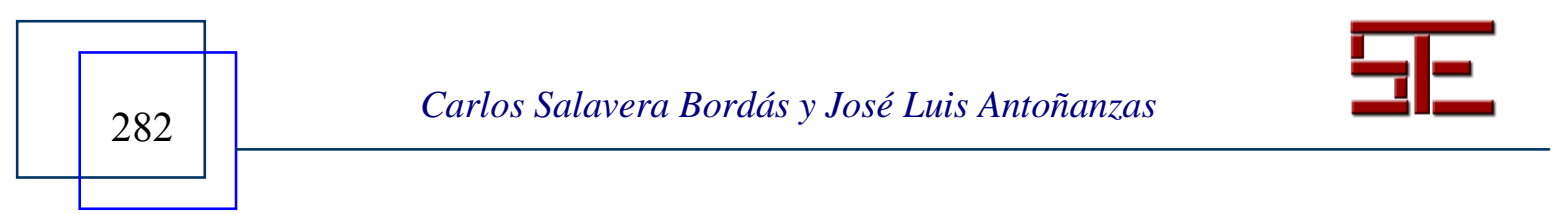




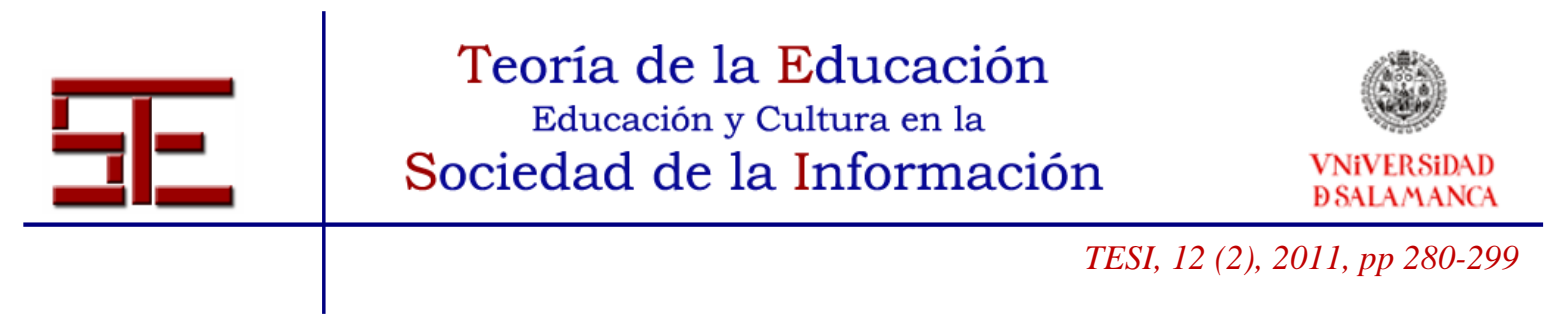

lado, un grupo más abierto con menor nivel de estructuración pero que igualmente trabajase aspectos cognitivos y de aprendizaje.

El objetivo era establecer si hay o no diferencia entre ambos grupos, si el grupo con mayor estructuración muestra mejoras significativas en los aprendizajes y sobre todo en las estrategias empleadas o si por el contrario era el grupo más abierto el que conseguía mejores estrategias de cara a los aprendizajes.

Se tomó como referencia el esquema de estrategias de aprendizaje de Philippe Meirieu, en el libro El gozo de aprender a tiempo de Gloria Medrano (1994), se filmaron ambos grupos en las secuencias lógicas de los aprendizajes y se analizaron interjueces con el objetivo de alcanzar la mayor objetividad posible. Se tomó un posicionamiento dicotómico entre las estrategias de aprendizaje con la idea de forzar a los interjueces a declinarse y obligar a hacer dinámico el proceso de toma de decisiones.

Distintos autores proponen investigar sobre las estrategias de aprendizaje, tanto a nivel global (Fernández, 2009; Monereo, 1997; Muria, 1994; Prichard; Stratford y Bizo, 2006; Soler; Núñez, González-Pienda y Álvarez, 2002), como aquellas que se establecen para la adquisición de las habilidades de comunicación específicamente (Salavera y Puyuelo, 2010; Van Nuland; Thijs; Van Royen; Van den Noortgate y Goedhuys, 2010).

Con la incorporación de las TIC, se produce una falla en la planificación de los alumnos, hay un exceso de monólogos, el discurso es pobre, cuesta un gran esfuerzo por parte del interlocutor-receptor conseguir nexos de unión entre las frases que den consistencia a la conversación, representando gran dificultad los métodos tradicionales utilizados con este fin de cambio de tema: al hilo y a saltos (Gómez; Sanz y Salavera, 2010).

Desarrollar habilidades de comunicación y estrategias de aprendizaje de estas mismas habilidades es el objetivo de ambos grupos de trabajo.

\section{MATERIAL Y MÉTODO}

El proyecto de investigación se diseñó como un estudio prospectivo y comparativo, con distribución aleatoria de los alumnos en dos grupos (experimental y control).

Ambos grupos cursaban estudios de Magisterio en la Facultad de Educación de Zaragoza. La participación fue voluntaria y se distribuyó a los sujetos según una entrevista previa.

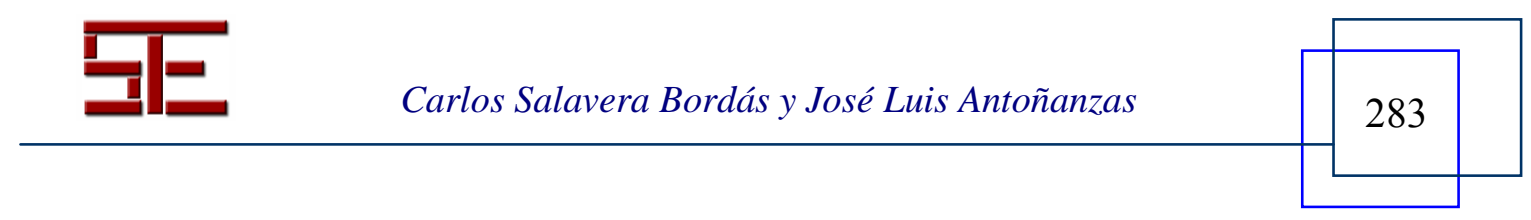




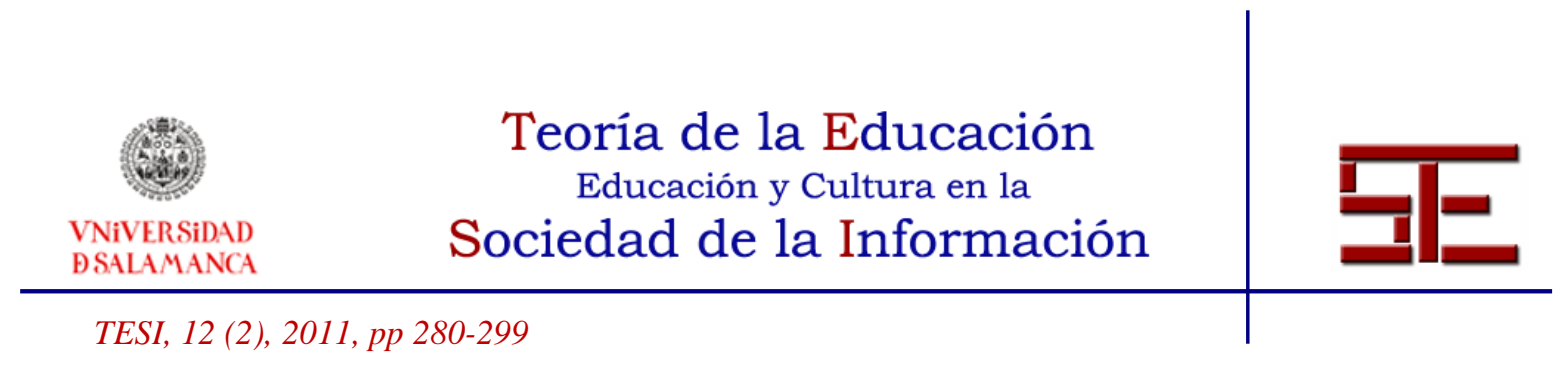

El grupo experimental formaba parte de un grupo (habilidades de comunicación) muy estructurado en cuanto a sesiones, contenidos y aprendizajes. El grupo control formaba parte de un grupo más abierto, dentro del área cognitiva, con una menor estructuración pero con aprendizajes referidos también en cuanto a lenguaje.

Para la evaluación de todos los aspectos referidos a aprendizajes, y más en concreto a las estrategias empleadas por los sujetos del estudio para la adquisición de las mismas, se utilizó la observación por medio de dos jueces entrenados anteriormente sobre los aspectos a observar.

Además se apoyó la investigación con filmaciones de vídeo para una mejor resolución y análisis de las estrategias que utilizaron. Se analizaron estas filmaciones con posterioridad, primero con los sujetos del estudio, viendo las observaciones que ellos mismos hacían sobre sí mismos y posteriormente por los jueces para resolver dicotómicamente las estrategias de aprendizaje utilizadas, siguiendo el esquema de Philippe Merieu.

Para la previa y establecer líneas base, se utilizaron test lingüísticos que nos proporcionaron información sobre aspectos verbales, en cuanto a aspectos sintácticos, fonológicos y semánticos previos a los aprendizajes que pretendíamos que adquiriesen los sujetos del estudio.

\section{Participantes}

Para la selección de la muestra se tomaron dos grupos de ocho alumnos, ambos estaban compuestos de seis mujeres y dos hombres y pareados en cuánto a edad. El grupo experimental configuraba un grupo de habilidades de comunicación y el grupo control conformaba un grupo de lenguaje mucho menos estructurado en cuánto a impartición de conceptos.

\section{Procedimiento}

Se evaluó a los alumnos en dos momentos: T0, que coincide con el momento de comienzo de los grupos de habilidades de comunicación y grupo de lenguaje, y en otro momento, T1, al final de los mismos.

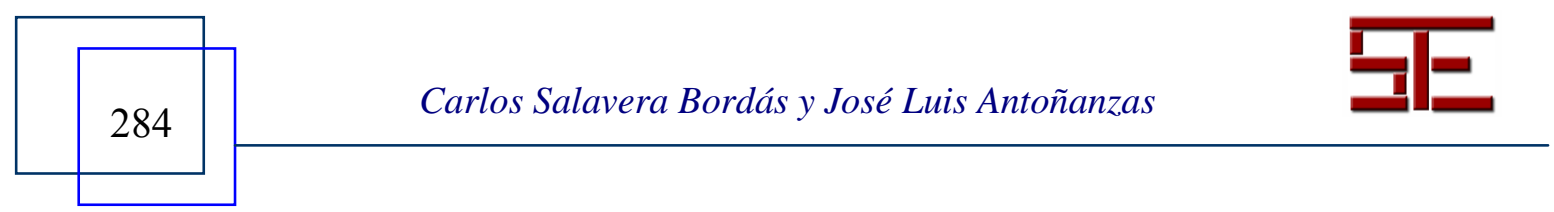




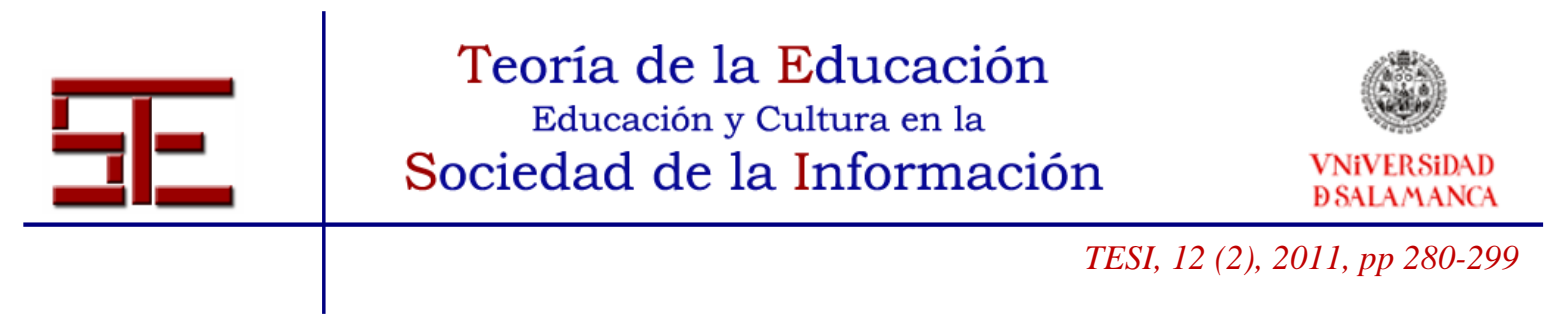

Como instrumentos de apoyo, se emplearon test relativos a lenguaje y que nos proporcionaron resultados comparativos intersujetos e intergrupos previos a la investigación.

Los test utilizados para la evaluación psicolingüística fueron el test de inteligencia WAIS, por ser un instrumento que nos indica el estado general global de los sujetos, así como de sus capacidades, además de una medida verbal tanto en el ámbito de coeficientes, como de índices e interrelación entre ellos.

También se utilizó el Test de Acentuación de Palabras (T.A.P.) de González Montalvo, que nos permite conocer capacidad verbal previa en los sujetos de estudio.

El test de personalidad EPQ-A de Eysenck fue utilizado para discriminar si había rasgos de personalidad que nos pudiesen sesgar el estudio.

Se pasó también una Escala de Habilidades Sociales, para identificar posibles problemas que estos problemas de funcionamiento en cuanto a estrategias de aprendizaje repercutían en los sujetos del estudio.

Estas valoraciones de los test se completaron con entrevistas individuales con los sujetos con los que ampliar la información y resolver pequeñas dudas en aspectos puntuales.

Ambos grupos tuvieron una duración de 16 sesiones, siguiendo la metodología observacional de Anguera. Se comparó a ambos grupos siguiendo directrices de estrategias de aprendizaje de Phillipe Merieu, en el libro El gozo de aprender a tiempo de Gloria Medrano.

Se valoró a los alumnos tras realizar una medición interjueces con entrenamiento previo de los mismos. Para ampliar esta valoración, se filmó a los alumnos de ambos grupos, con lo que permitía resultados más estables y de mayor fiabilidad.

\section{RESULTADOS DE LA INVESTIGACIÓN}

La edad media de la población del estudio se sitúa en 20.20 años, con un rango 18-32 y una desviación estándar de 2.030.

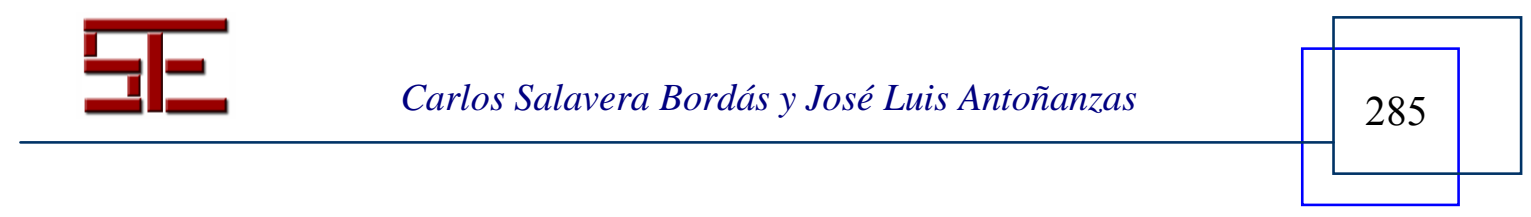




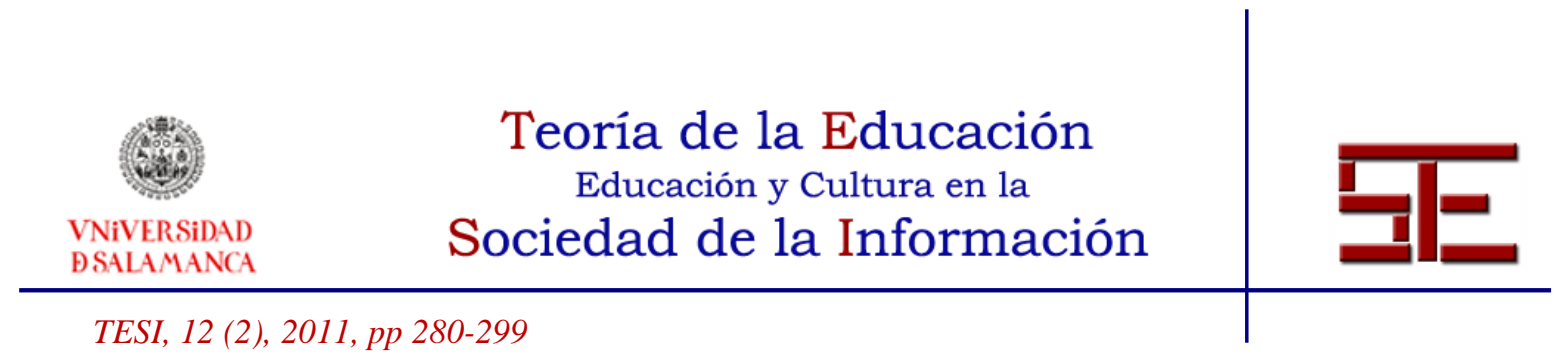

El Coeficiente Verbal es de 106.67 de media, con un Coeficiente Manipulativo de 96.56 y un Coeficiente Intelectual de 102.61. No hay diferencias significativas pues, en cuanto a edad o coeficientes intelectuales entre ambos grupos.

Tampoco hay diferencias en cuanto a personalidades previas, medidas con el test de personalidad EPQ-A, que pudiesen influir en cómo se desempeñan funcionalmente.

En el test que medía la capacidad verbal previa de los sujetos (TAP de González-Montalvo), no se encontraron diferencias entre ambos grupos.

Si tenemos en cuenta las estrategias de aprendizaje señaladas por Philippe Merieu, tenemos resultados como que los útiles de aprendizaje, utilizados no por nosotros sino por los participantes en el estudio, cuando se les pidió que explicasen algún tema para todos, tenemos:

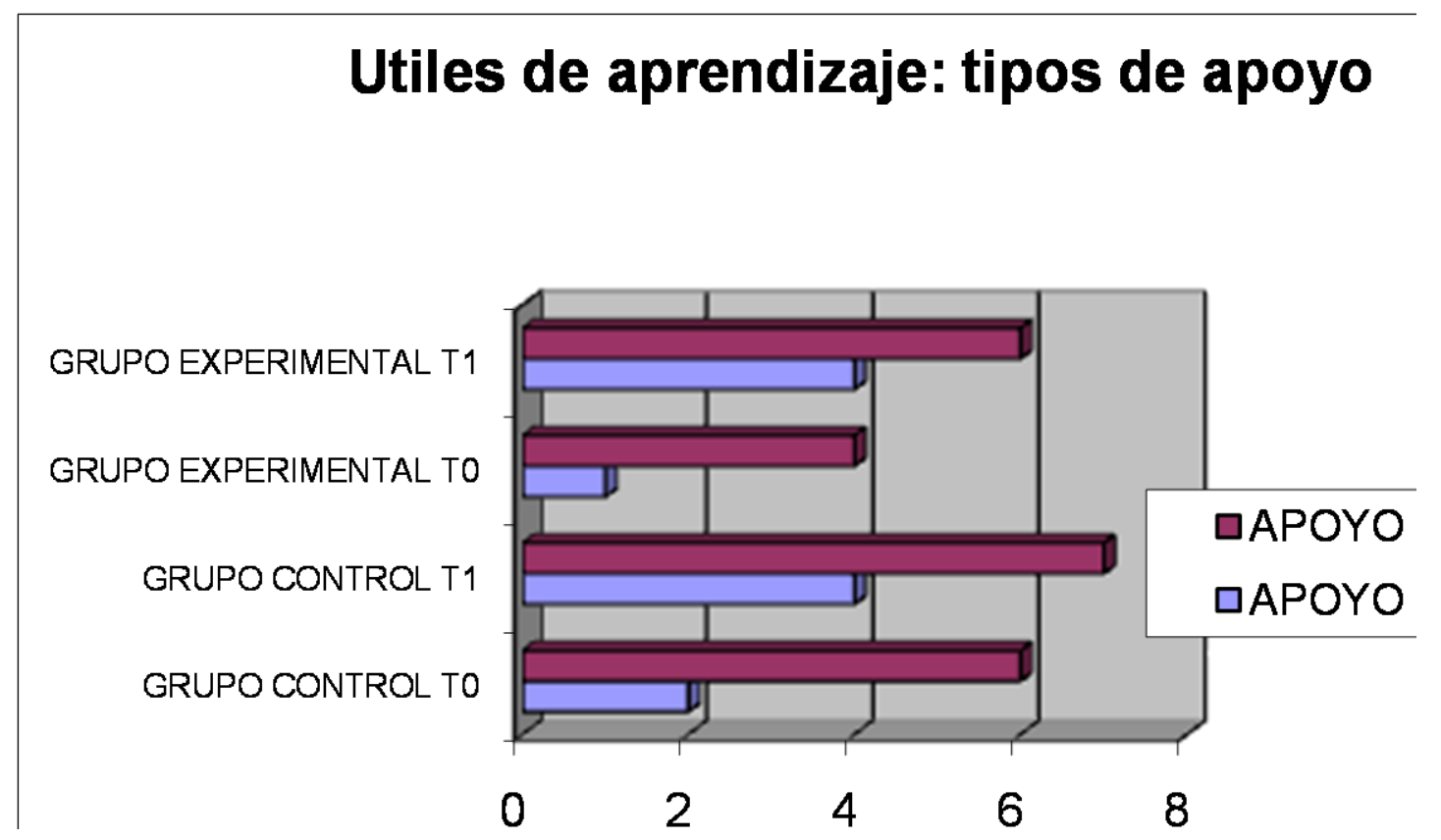

Como vemos, en el grupo experimental en un principio hubo integrantes que no supieron resolver cómo realizar la explicación del tema para sus compañeros, sin embargo, una vez explicado cómo podían hacerlo utilizaron no sólo el apoyo escrito, sino también el verbal con una buena ejecución de la tarea. En el grupo control en un

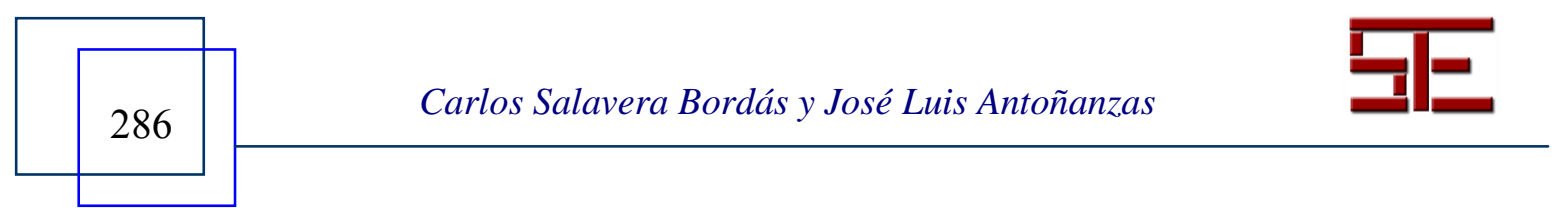




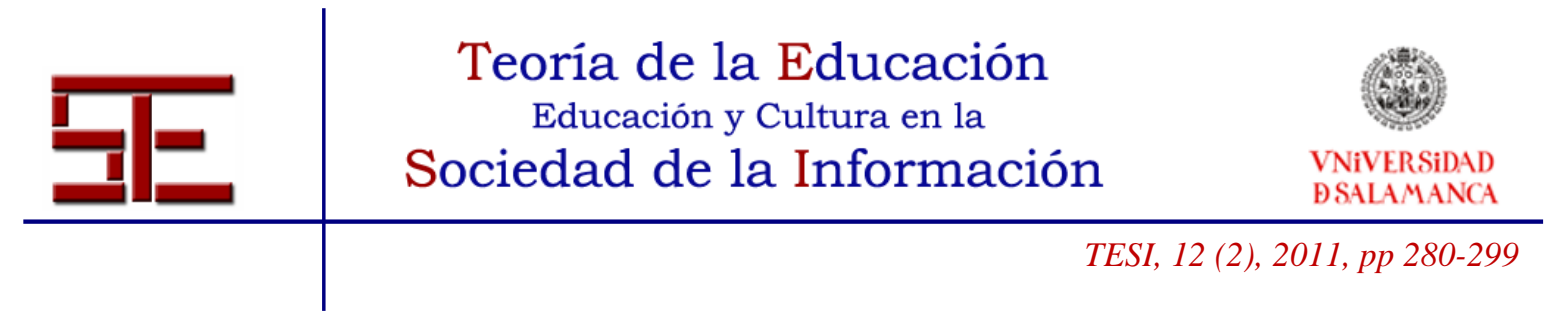

principio las puntuaciones son algo mayores en el momento T0, para en el momento T1 ser muy similares a las del grupo experimental.

En el momento del analizar cómo realizan el abordaje de sus compañeros, en el grupo experimental no afrontan bien en el T0 cómo realizarlo, mejorando espectacularmente cuando avanzado el programa se les han indicado pautas de cómo hacerlo. En el grupo control, obtienen resultados algo mejores en el momento T0, para en el momento T1, sus puntuaciones ser idénticas a las del grupo experimental.

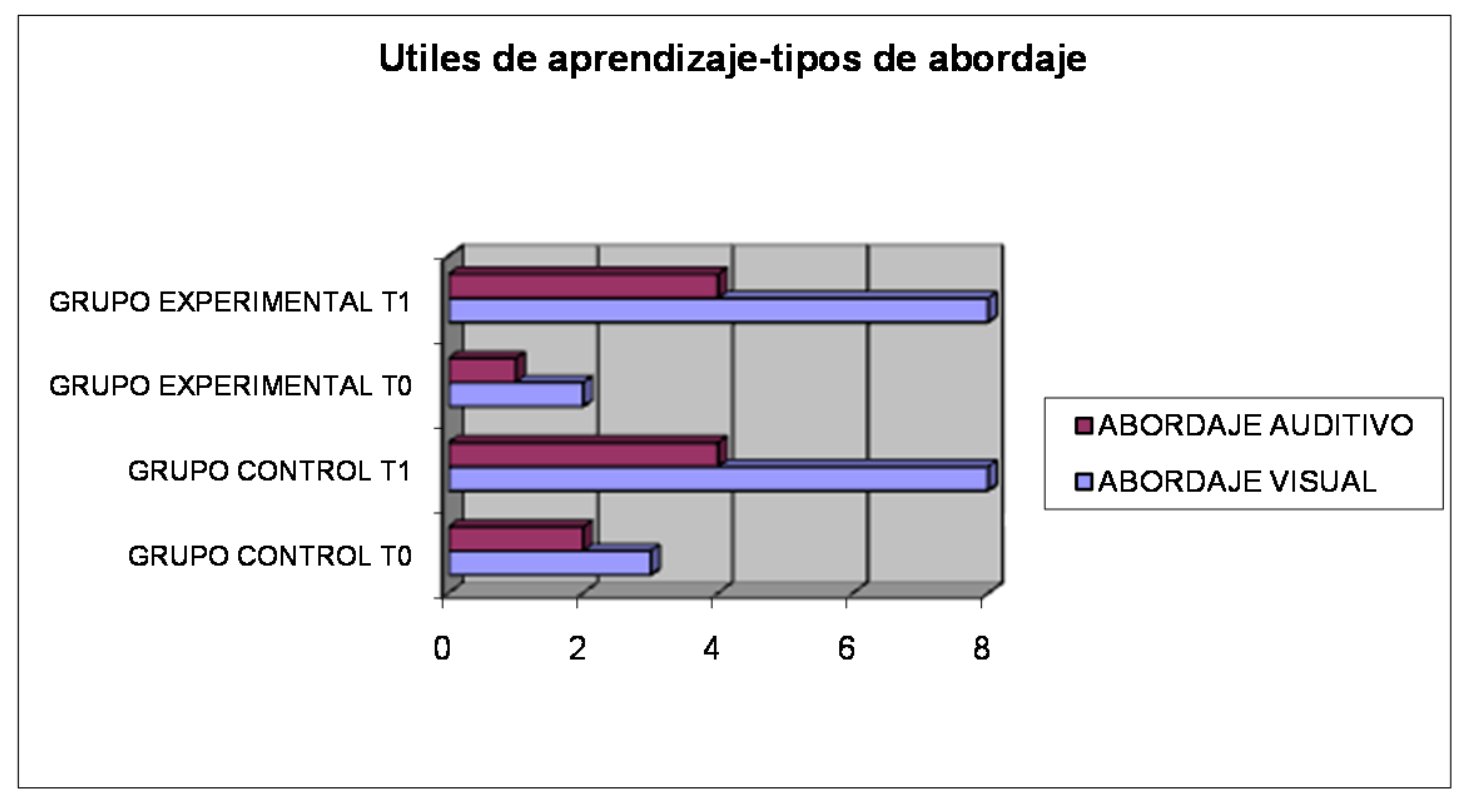

Las aproximaciones, en el grupo experimental en el momento T0, se vio que era una situación que difícilmente podían resolver, para en el momento T1, al final del programa, utilizar no sólo el contacto directo con los objetos, sino también la representación simbólica, mezclando las dos con mucha efectividad.

En el grupo control, en un principio no emplearon la aproximación de representación, y poco la manipulativa. En el momento T1 utilizaron todos los participantes la representación simbólica y se apoyaron en la aproximación manipulativa.

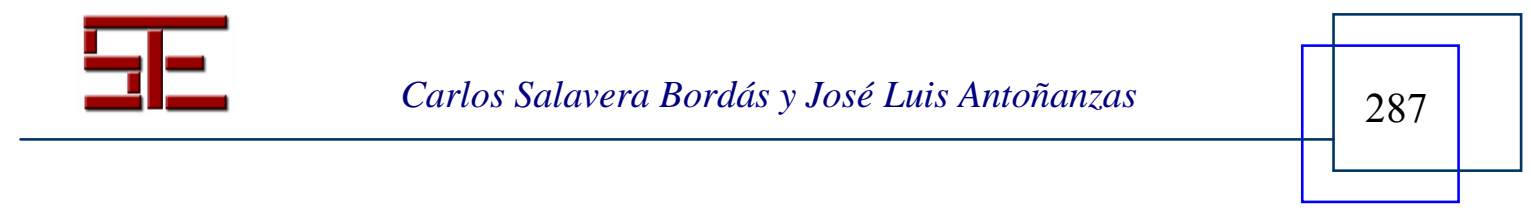




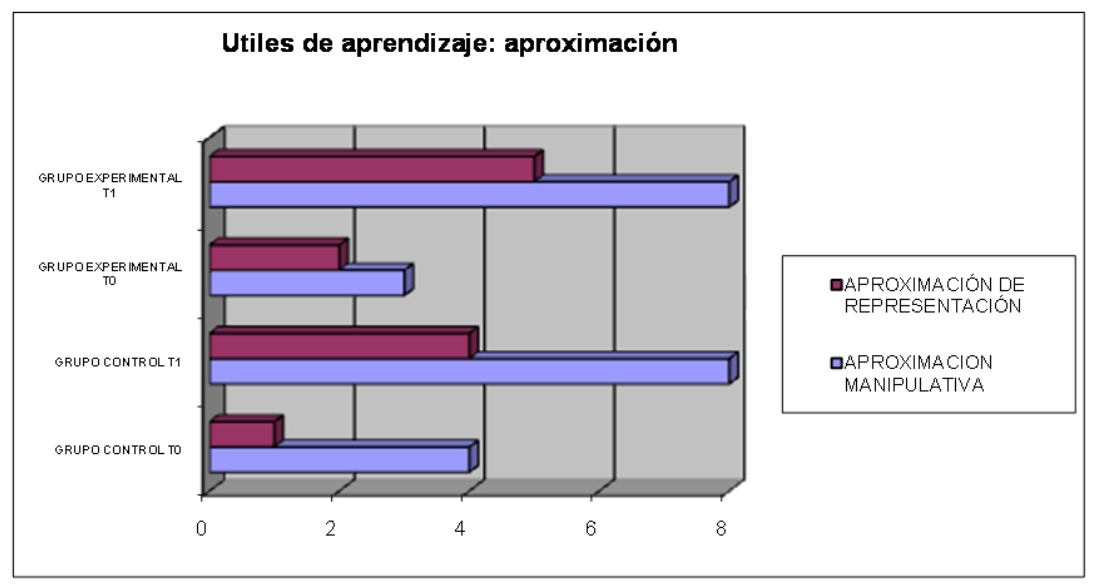

En el aspecto de cómo se realizaron las aproximaciones, tenemos en el grupo experimental que desde el principio utilizaron más la aproximación global al tema, con puntuaciones altas también en cuanto a la posibilidad de aproximación global. En el grupo control, se apoyaron menos en aspectos de aproximación, optando más por una explicación o por el silencio cuando no consiguieron explicar algo.
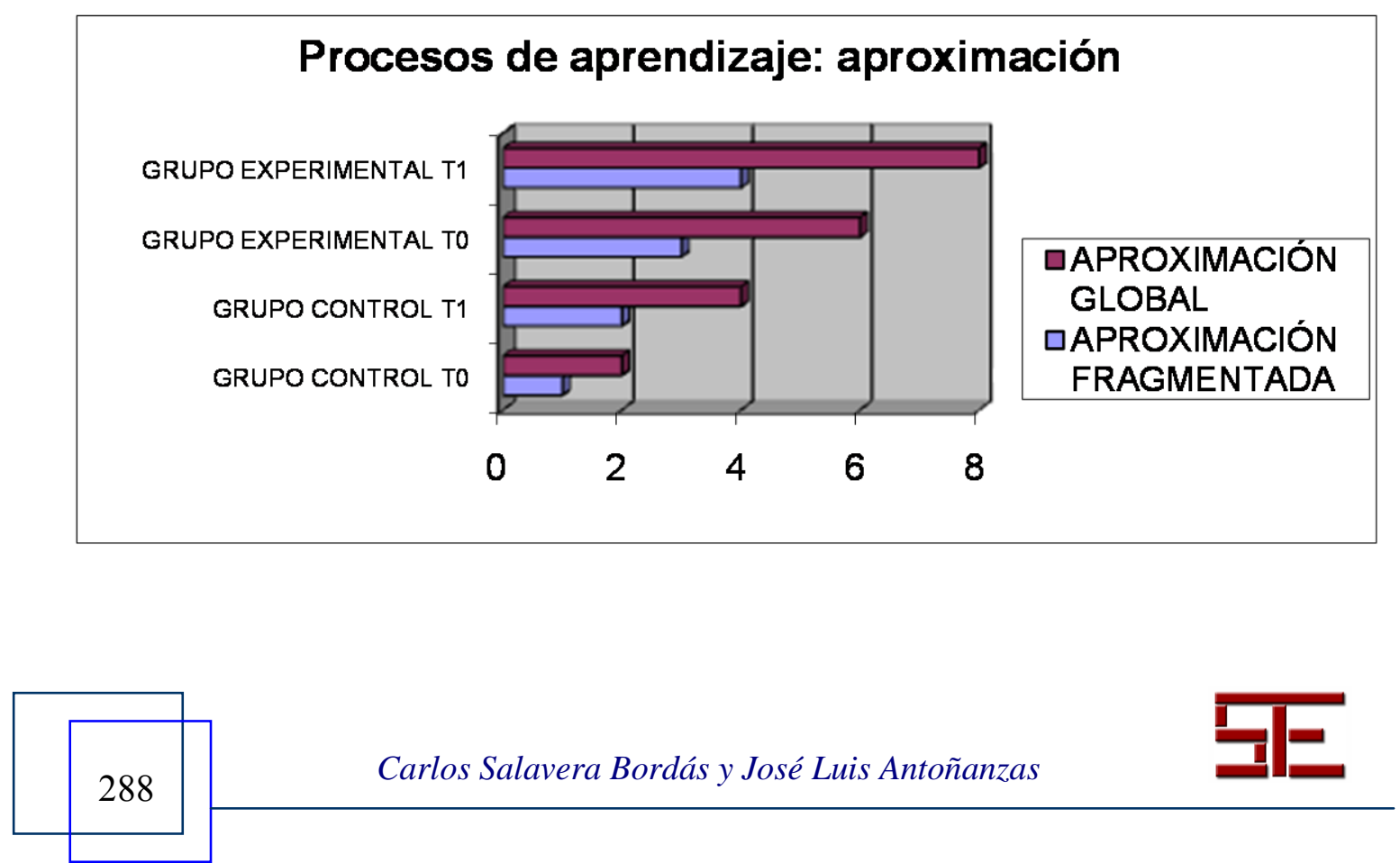


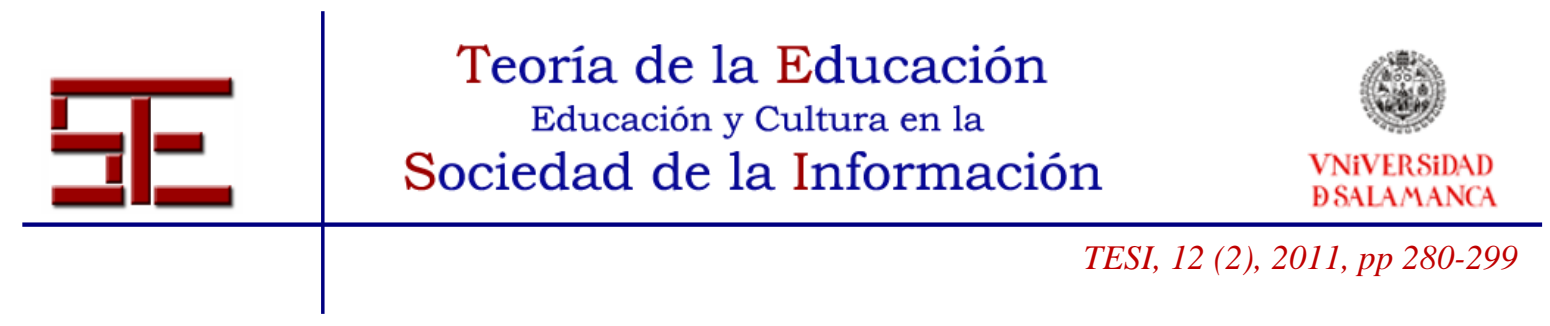

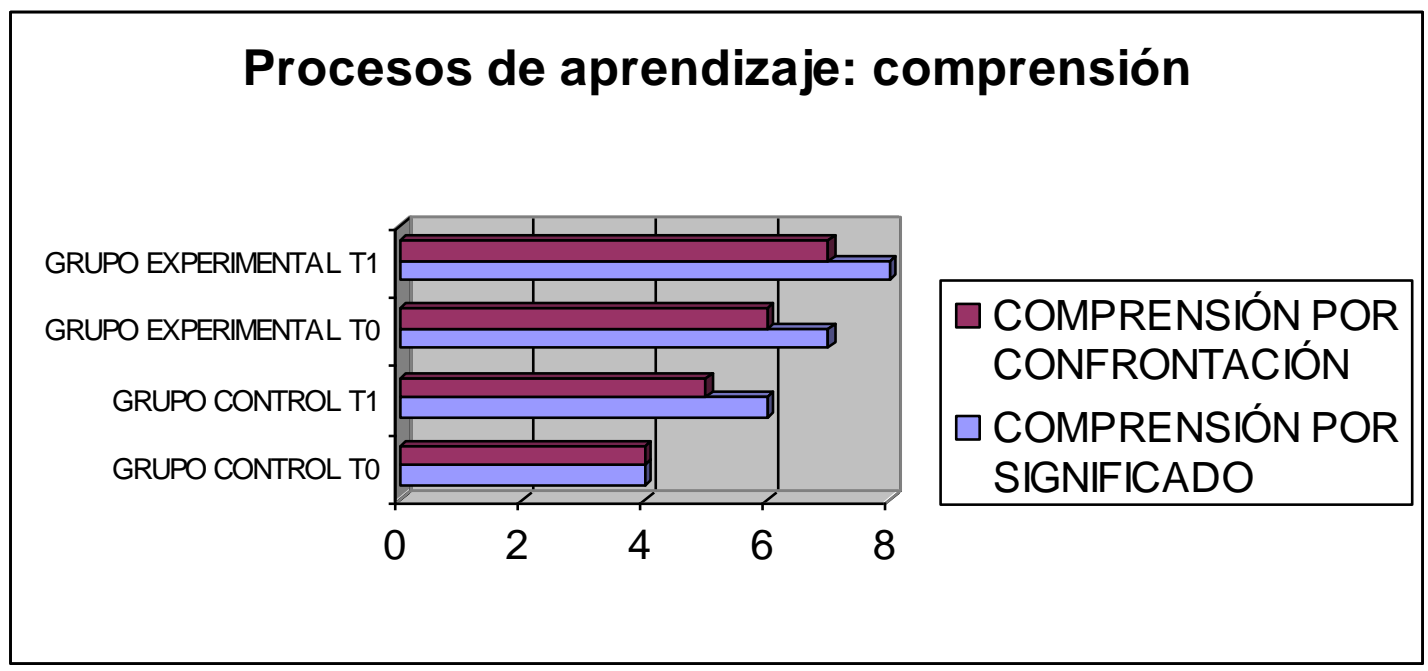

En cuanto a procesos de aprendizaje, si analizamos cómo se ha realizado el proceso de comprensión de los nuevos conceptos, vemos que en el grupo experimental en el T0 hay un mayor nivel de comprensión claramente observable, incrementándose tanto las puntuaciones de comprensión por significado, en el que se aprende primero el principio y el sentido de algo, aunque también aumenta la comprensión por confrontación, donde se aprende por contraste.

En el grupo control, tanto en T0 como en T1 se obtienen puntuaciones menores que en el caso del grupo experimental en los mismos conceptos.
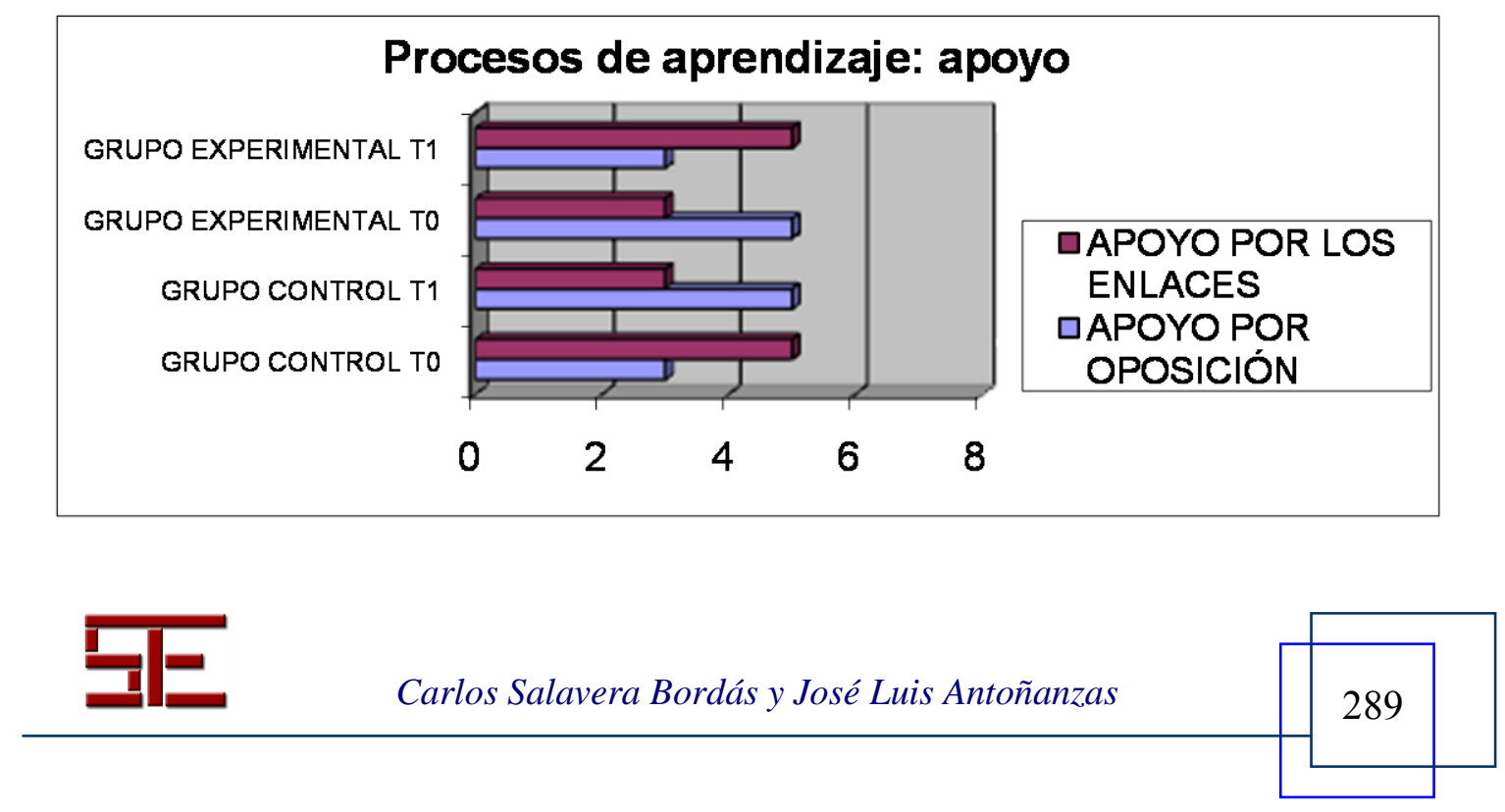


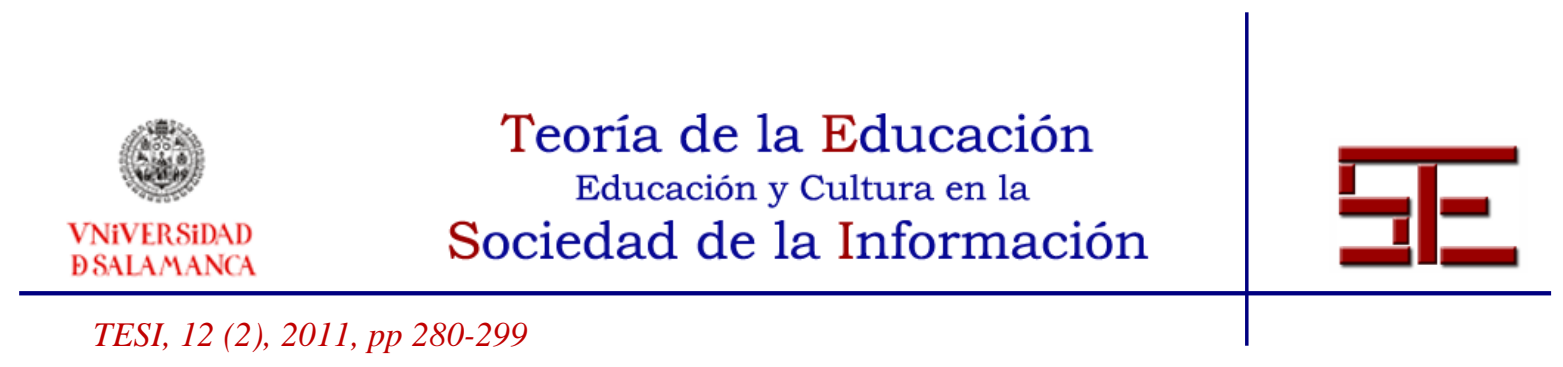

En cómo realizan los procesos de aprendizaje en cuanto a apoyos, vemos que en el grupo experimental, en un principio (T0), se alcanza mayor puntuación en el uso de apoyos por oposición, donde lo hacen por diferencias. En T1 se incrementa el uso de apoyos por los enlaces, buscando nexos de unión entre conceptos.

En el grupo control, en T0 hay un mayor uso de apoyo por los enlaces por encima del uso de apoyos por oposición, sin embargo, en T1 este fenómeno se invierte, dándose un mayor uso de apoyo por oposición, buscando las diferencias entre conceptos.

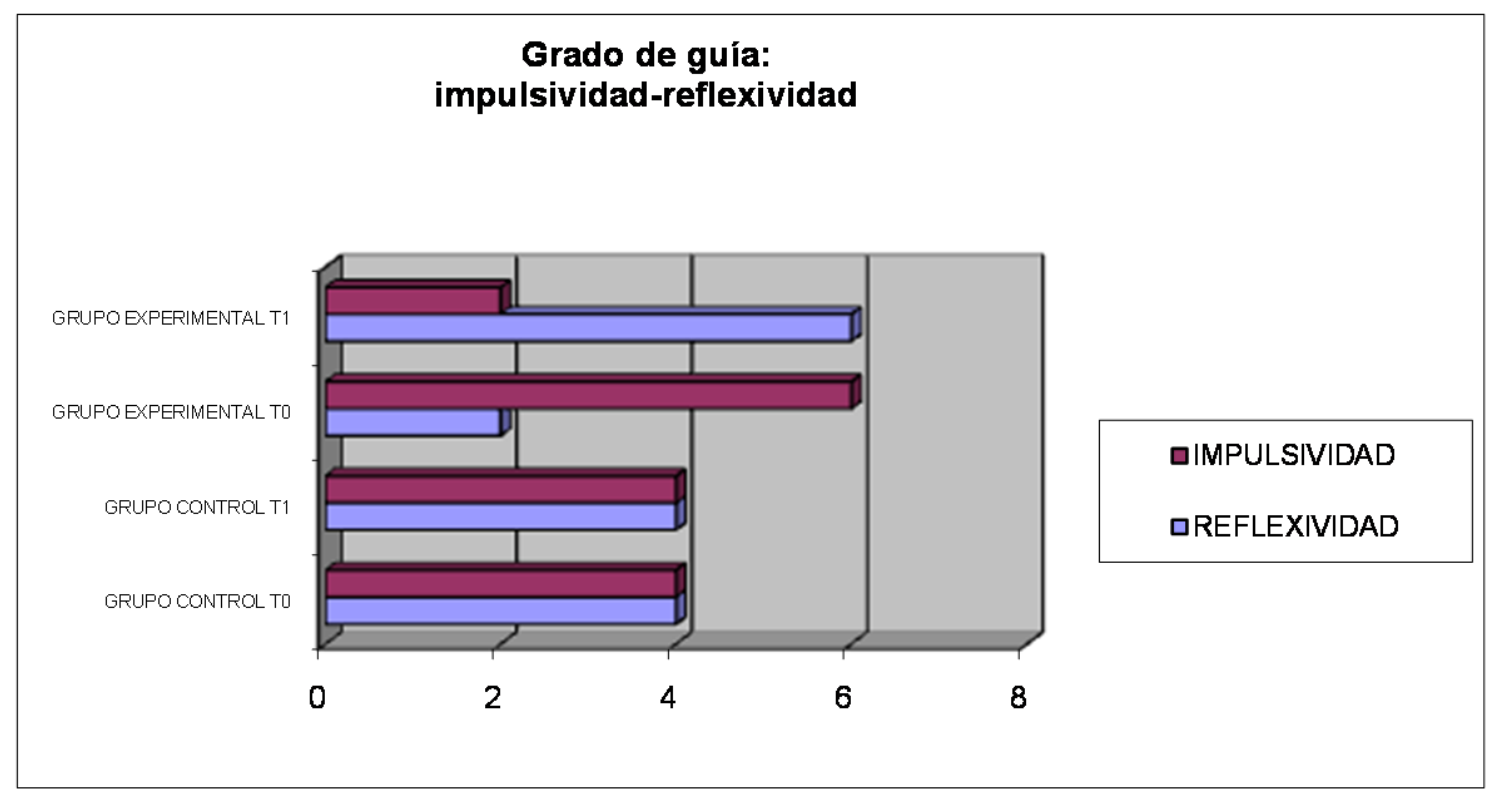

En el grupo control no hay diferencias entre impulsividad, conceptualizada como la aproximación inicial global y superficial que se precisa y concreta a posteriori, y reflexividad, tomada como una progresión lenta y meditada.

Donde sí se presentan diferencias significativas es en el grupo experimental, en este grupo se da un aumento de reflexividad en el mismo grado en que disminuye la impulsividad.

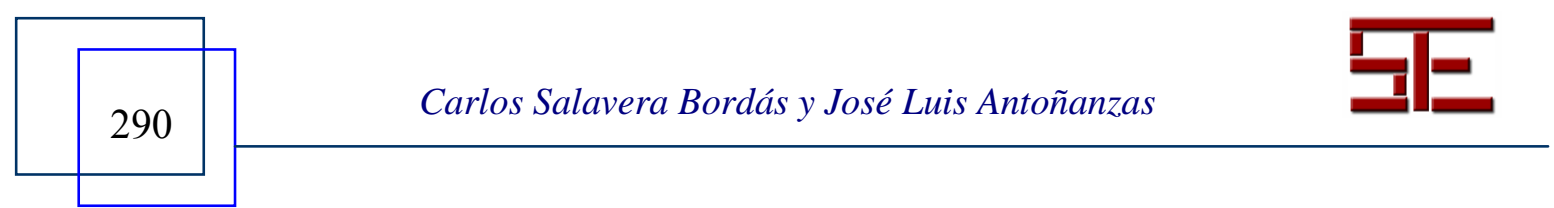




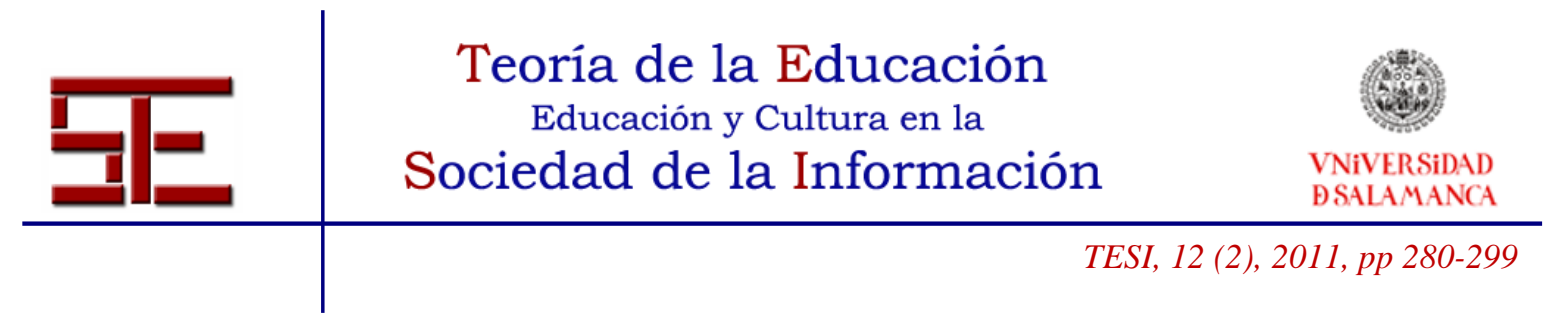

\section{Grado de guía: necesidad}

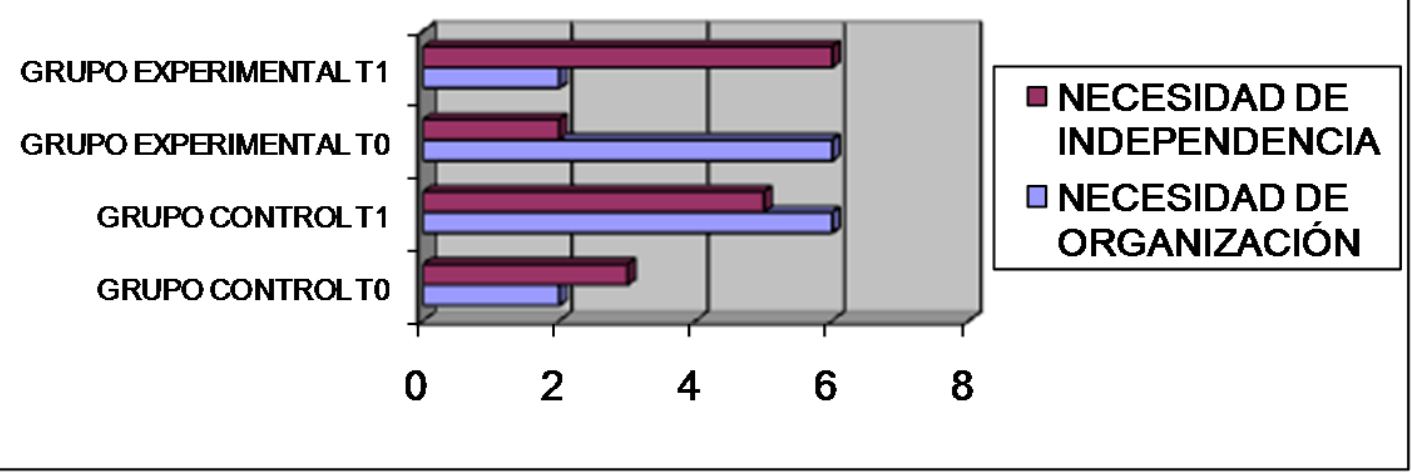

En el tema del grado de guía empleado, tenemos que en el grupo experimental hay una disminución de la necesidad de organización (apoyos de recuerdo, guía y orientación) y un aumento de la necesidad de independencia (progresión organizada libremente por el sujeto que aprende).

En el grupo control hay un incremento de la necesidad de organización y de la necesidad de independencia simultáneamente.

Este aspecto podemos calificarlo de positivo, sobre todo en cuanto al incremento en ambos grupos de la necesidad de independencia, lo cual dice de la primera adquisición de estrategias de aprendizaje, aun no siendo ellos mismos conscientes de esto.
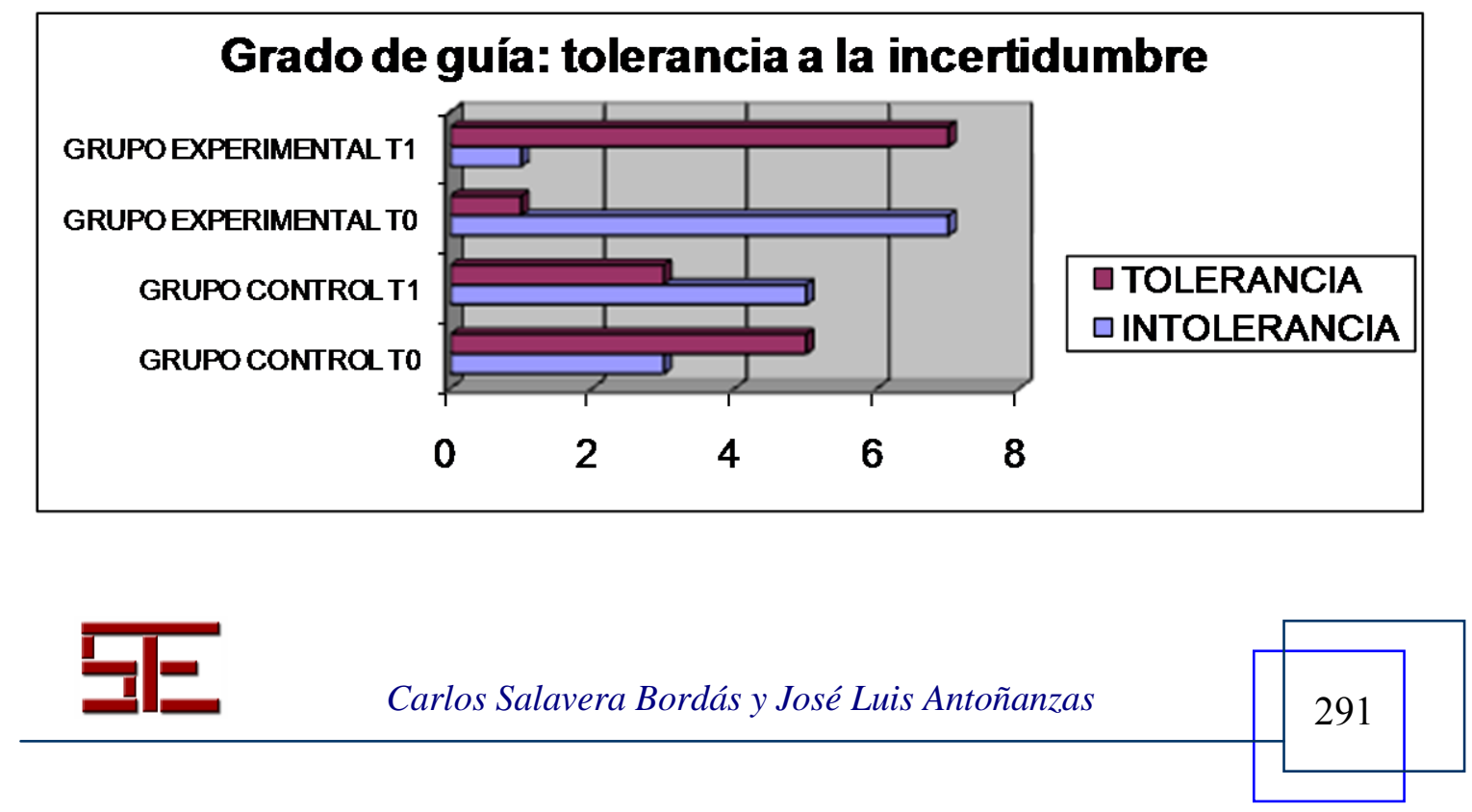


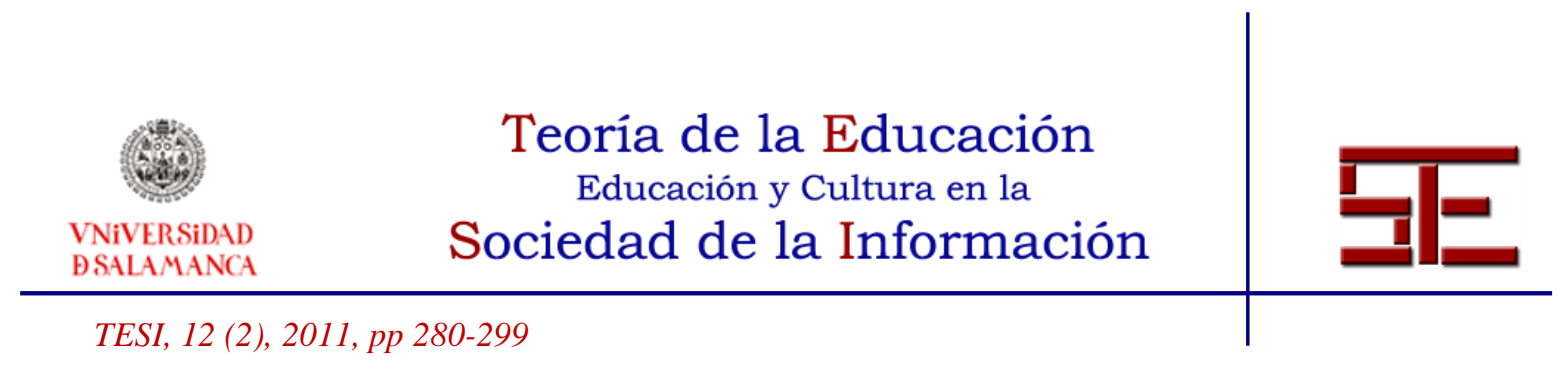

Si contemplamos, como dice Merieu, la tolerancia a la incertidumbre como el uso de una propuesta abierta para que el sujeto descubra los objetivos a partir de formulaciones sucesivas y la intolerancia como el uso de normas, planes, proyectos y objetivos delimitados claramente, obtenemos observaciones como que en el grupo experimental hay un incremento en $\mathrm{T} 1$ de la tolerancia usando aproximaciones sucesivas. En el grupo control hay un pequeño descenso de la tolerancia a la incertidumbre y un pequeño aumento de la intolerancia en T1.

En el ámbito de la inserción socioafectiva, si analizamos el subapartado de la implicación, vemos que Merieu conceptualiza esto en dos términos: distanciamiento como la capacidad de distanciamiento afectivo para organizar fría y racionalmente la situación e implicación como la necesidad de integrarse afectivamente en la situación.

En el grupo experimental hay una disminución del distanciamiento y un incremento de la implicación conforme avanza el tiempo.

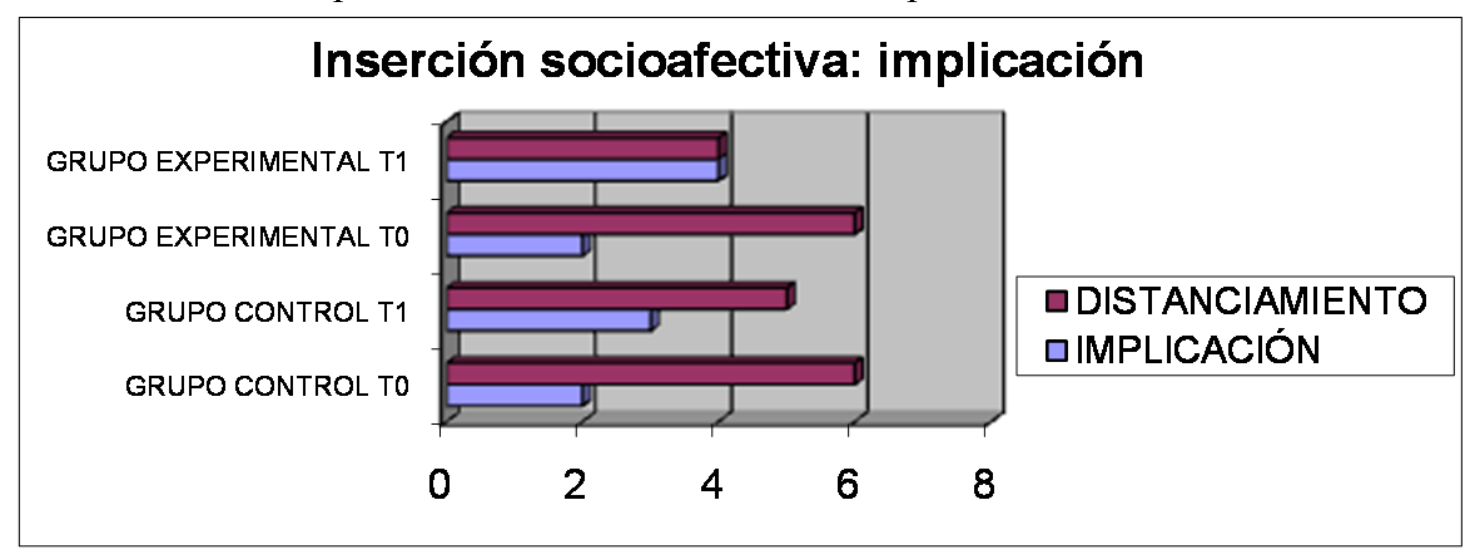

En aspectos como la dependencia o independencia de las relaciones sociales para el establecimiento de aprendizajes, vemos que en el grupo experimental en T0 la puntuación es un poco mayor en cuanto a independencia (necesidad de aislamiento social para la actividad del aprendizaje) que a dependencia (necesidad de apoyo de los demás para el proceso de aprendizaje).

En T1 ambas variables están igualadas, reflejo del proceso de cooperación que se produjo en este grupo. En el grupo control, se produce el fenómeno inverso, en T0 dependencia e independencia son igualmente utilizadas, para en T1 ser mayor la dependencia, apoyándose más en sus compañeros para el proceso de aprendizaje.

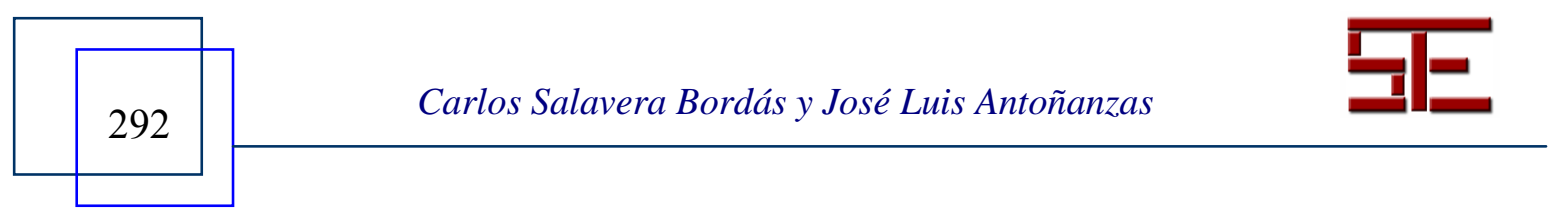




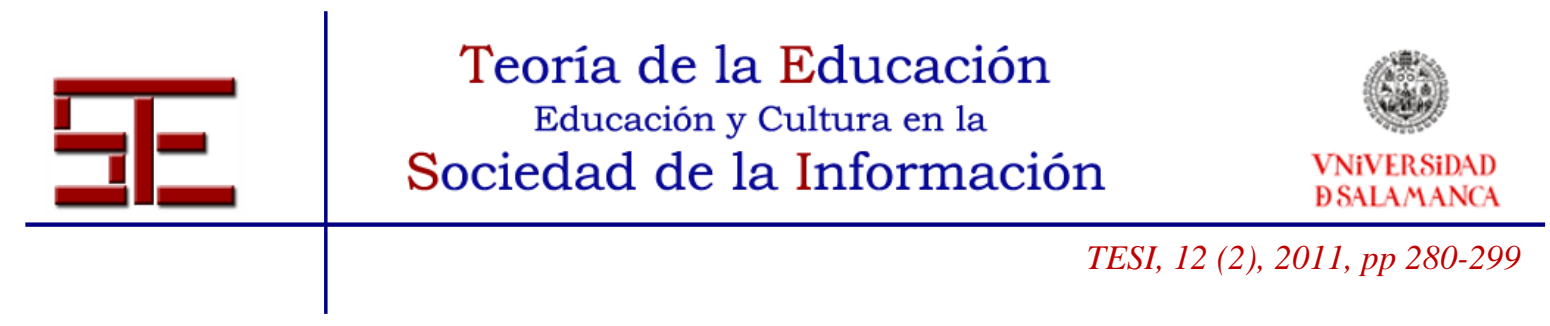

\section{Inserción socioafectiva: dependencia}

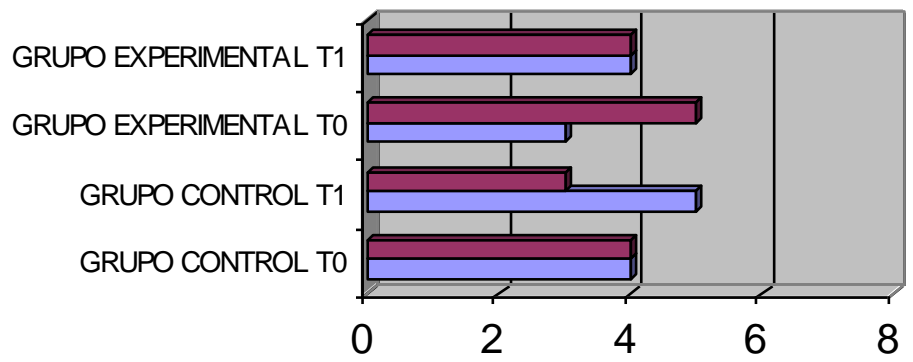

INDEPENDENCIA RELAC. SOC.

$\square$ DEPENDENCIA

RELAC. SOC.

En aspectos de la inserción socioafectiva como convergencia, referida al apoyo en el aprendizaje en cuestiones distintas a las de la situación en la que se aprende y búsqueda de nuevos enfoques, y divergencia, referida a la búsqueda de respuestas relacionadas con lo aprendido y en coincidencia con el enfoque establecido, vemos que en el grupo experimental en T0 se da una mayor convergencia por encima de la divergencia, para en el tiempo T1 igualarse ambos.

En el grupo control en T0 hay un uso por igual de ambas estrategias, para en T1 ser mayor el uso de la divergencia como método de aprendizaje.

\section{Inserción socioafectiva: convergencia}
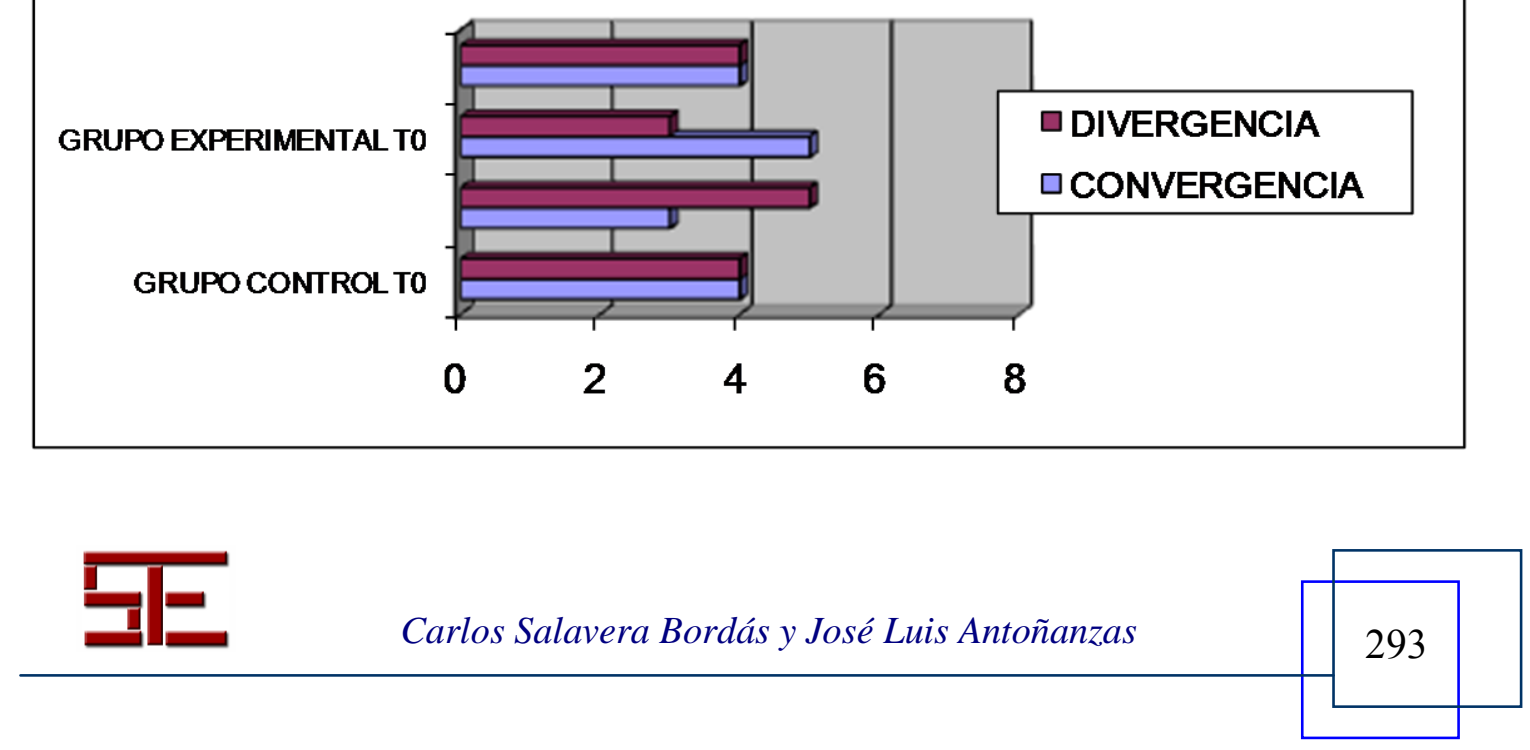


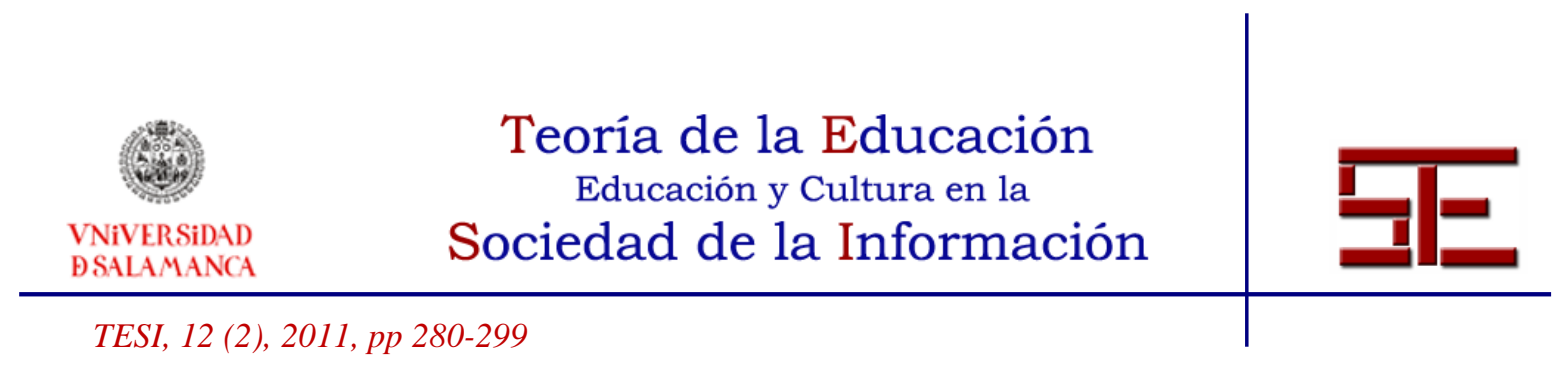

En la distribución del tiempo, en cuanto a tipo de trabajo tenemos dos posibilidades: el uso de la impulsividad, conceptualizada como el uso de respuestas rápidas e inmediatas, y la secundariedad, tomada como el empleo de una respuesta retardada por la reflexión previa.

En este apartado, vemos en el grupo experimental en T0 como el uso de secundariedad e impulsividad obtienen resultados idénticos, para en T1 darse un gran aumento de la secundariedad y un descenso en la impulsividad, fruto del trabajo estructurado con el grupo.

En el grupo control, en T0 obtenemos resultados exactamente iguales, para en T1 incrementarse la secundariedad, pero con resultados menos espectaculares que en el grupo experimental.

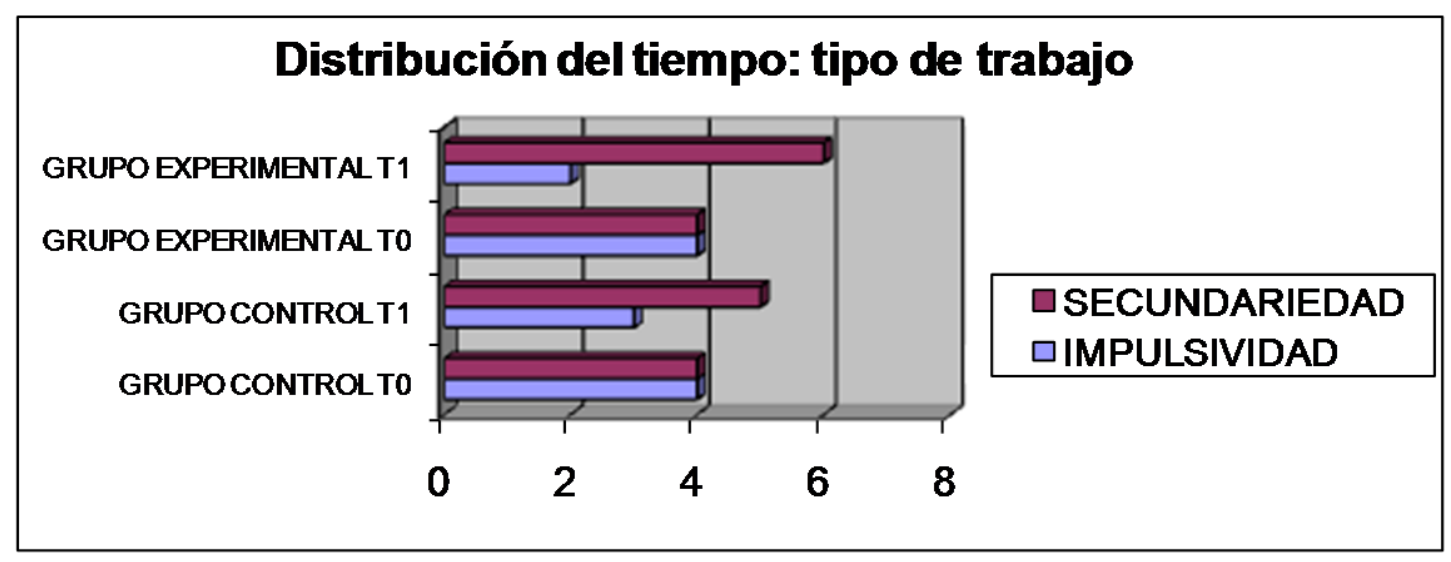

En la distribución del tiempo en cuanto a cómo se recoge la información, si este proceso lo ejercen los sujetos mediante una integración progresiva de la información o si por el contrario lo hacen mediante una recogida previa de la información, esto es, antes de actuar el sujeto busca toda la información necesaria (poca información y pocos errores) obtenemos resultados como que en el grupo experimental en T0 vemos que hay sujetos que no ejecutan bien esta función, en T1 una vez realizado el programa vemos que aumenta el proceso de recogida de información en ambos aspectos, tanto integrando información nueva como por la recogida previa de esta información.

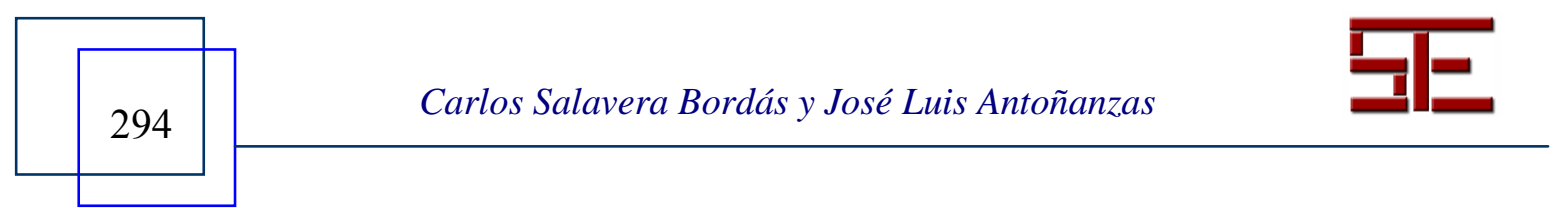




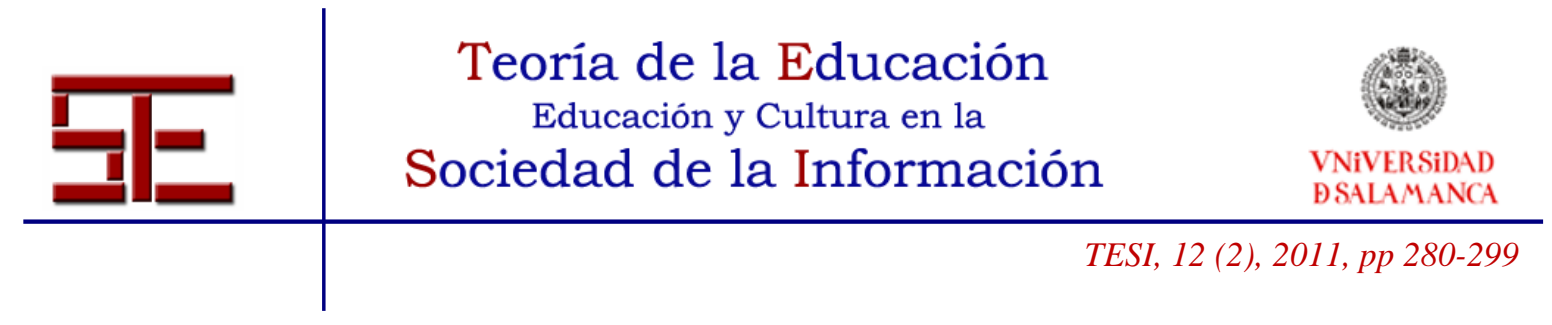

En el grupo control, obtiene resultados menores tanto en T0 como en T1 que el grupo experimental con incrementos muy semejantes a los del grupo experimental.

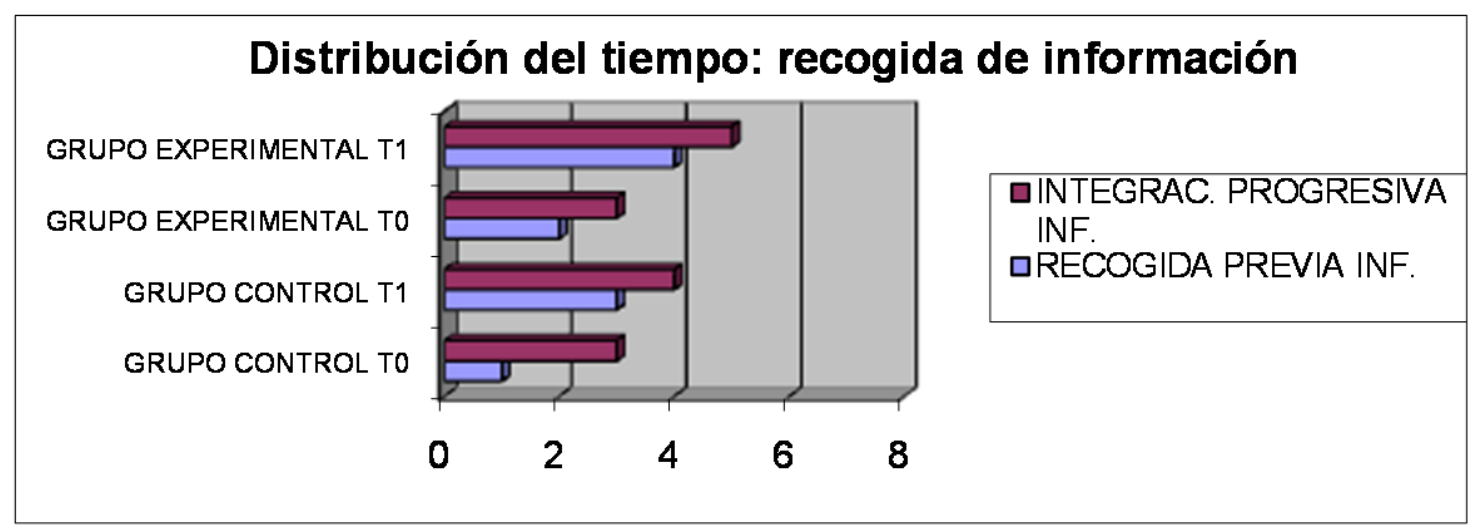

En la distribución del tiempo referido sobre si el trabajo es segmentado por el sujeto, trabajo en cortos períodos de tiempo y cambios frecuentes de actividad o por el contrario es un trabajo segmentado, centrado largamente en la misma tarea, en el grupo experimental en T0 predomina el trabajo segmentado sobre el continuado, para en T1 igualarse ambas puntuaciones.

En el grupo control en T0 hay una diferencia espectacular entre trabajo segmentado y continuado, predominando el primero, para en T1 igualarse ambos tipos de trabajo como estrategia de trabajo para el aprendizaje.
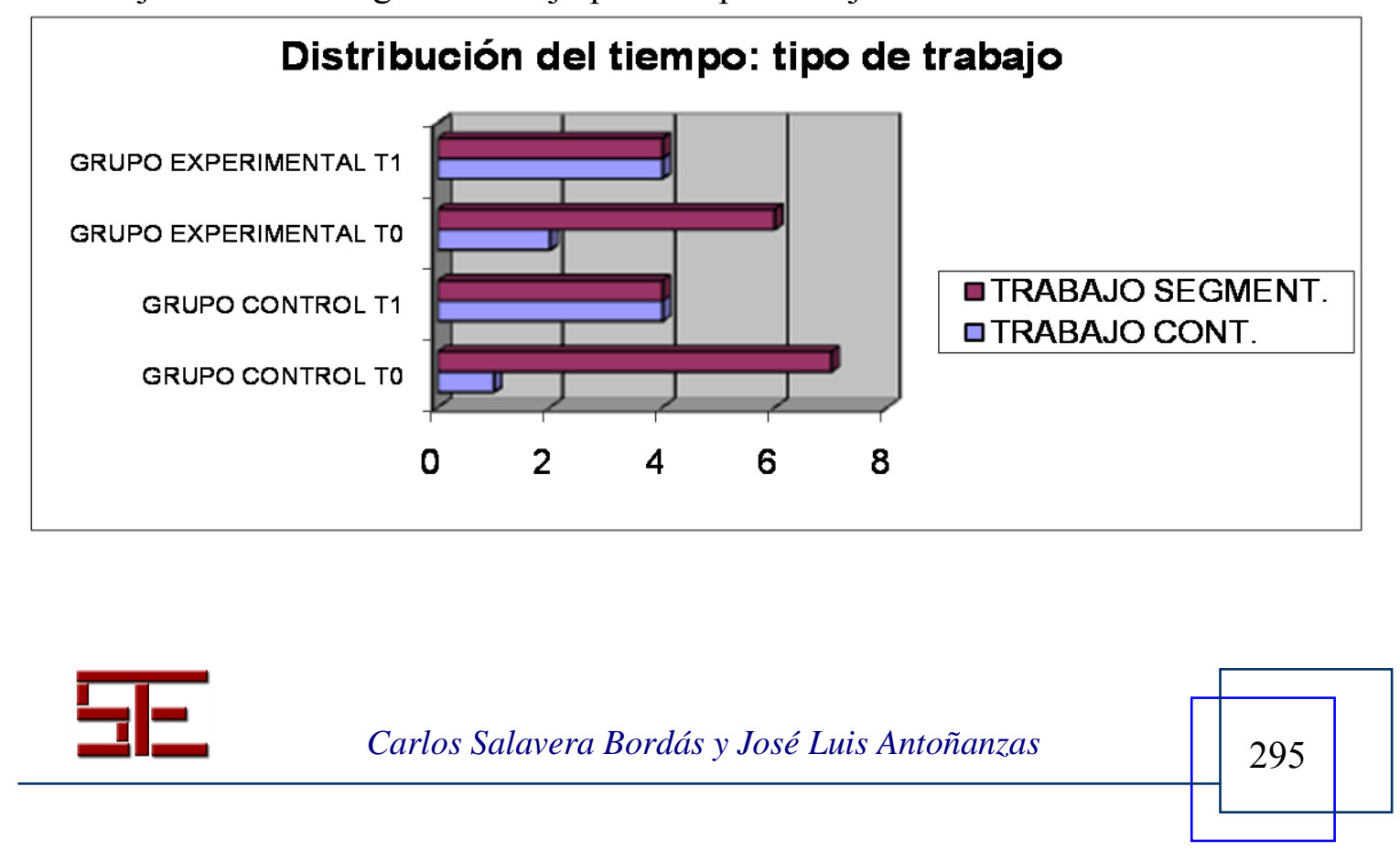


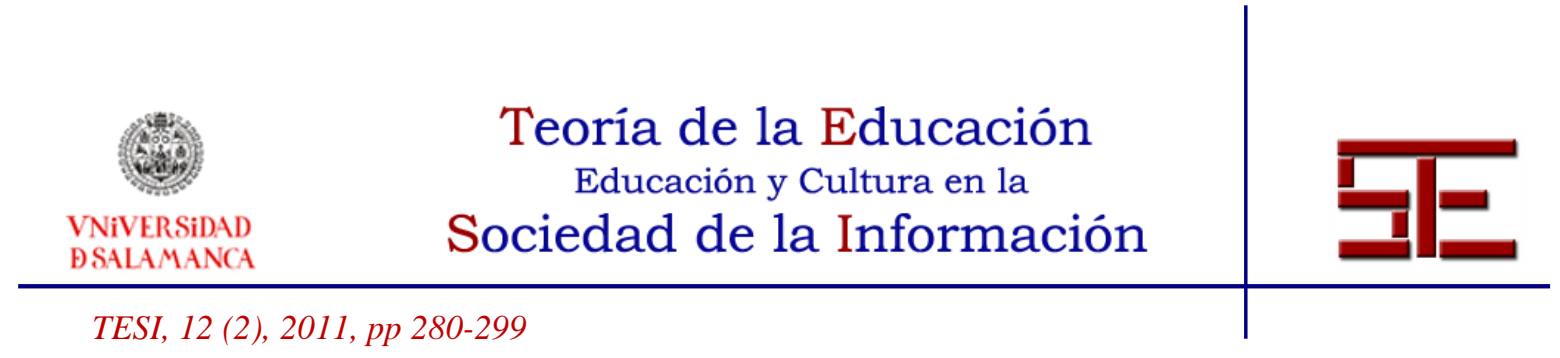

\section{DISCUSIÓN Y CONCLUSIONES}

Como primeras conclusiones, vemos que en los participantes del grupo que por ahora han participado en ambos programas, 16 en total, se ha incrementado la adquisición de estrategias de aprendizaje y ejecución de las mismas.

Además, han desarrollado mecanismos de asertividad y se ha desarrollado un buen clima grupal entre los participantes y con los profesores que intervinieron como terapeutas.

Se observa la necesidad de un programa en el que no sólo se entrene al sujeto en ejercicios repetitivos de formulación de pregunta-respuesta, sino que además se incentive el hablar por hablar, del tema que sea, con otros y la búsqueda propia de soluciones ante problemas cotidianos que acontecen a esta población tiene grandes efectos beneficiosos, sobre todo, en aspectos de adquisición de estrategias de aprendizaje y la adquisición de nuevos aprendizajes o recuerdo de anteriores aprendizajes, que el sujeto ya poseía pero no demostraba o practicaba, redundando en las competencias profesionales como docentes (Andreu et al., 2009; Renzulli, 2010).

Además de ser muy beneficiosos en cuanto a incremento de autoestima y de mejora en la relación entre ellos y como futuro docente.

En el grupo experimental que ha realizado un programa mucho más estructurado y delimitado, los resultados nos parecen alentadores. Ha aumentado la conciencia de adquisición de nuevos aprendizajes y, lo que es más importante, la demostración y manifestación de los mismos. En este grupo aumentó la comunicación entre ellos, afirman que se sienten más capacitados para iniciar y mantener conversaciones, para rechazar peticiones, así como para formular peticiones (pedir favores) consideradas como razonables, defender sus derechos, mostrar expresiones de afecto, expresar su opinión, hacer frente a las críticas o expresar su desacuerdo con algo / alguien.

Como primeras conclusiones del estudio, tenemos que no hay diferencia entre ambos grupos en cuanto a coeficientes intelectuales intergrupos.

Ambos grupos (habilidades de comunicación más estructuradas en cuanto a contenidos y lenguaje con contenidos de carácter más semiestructurados) presentan puntuaciones en todo caso positivas, demostrando que en los dos se producen

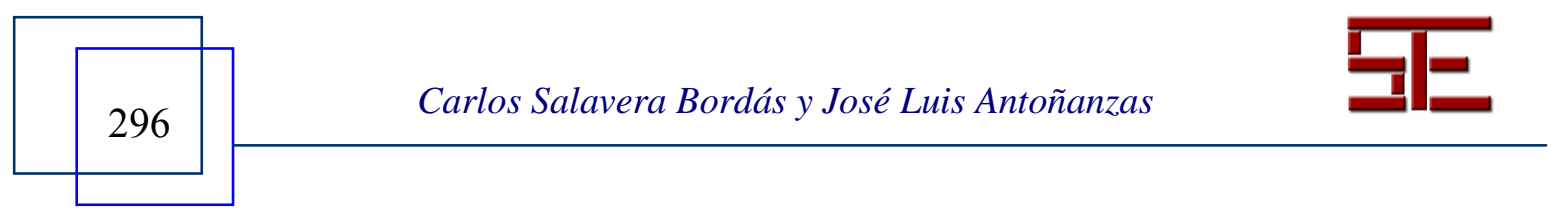




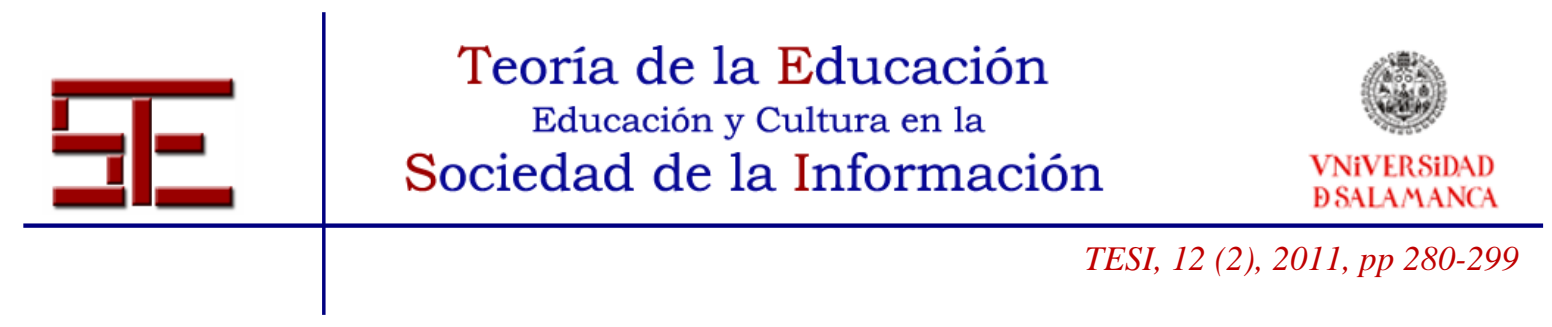

aprendizajes, que en algunos aspectos son similares y en otros son más favorables en el caso del grupo experimental.

Si tomamos como referencia los coeficientes verbales, tampoco las diferencias en ningún momento son significativas. En aspectos de coeficientes manipulativos tampoco hay diferencias.

En lo que se refiere a nivel verbal previo, medido por el Test de Acentuación de Palabras (TAP), no hay diferencias significativas entre ambos grupos, lo cual indica que su ajuste premórbido es similar (Ramos ; Manga, 1991).

En el test de personalidad EPQ-A, las diferencias no son significativas, que pudiesen señalar mejor o peor disposición de cara a los programas de adquisición de aprendizajes, en función de la personalidad del alumno.

Hay diferencias muy significativas en la Escala de Habilidades Sociales, en lo que se referiría a desempeño social de los sujetos, y no hay que olvidar que el lenguaje y el uso que se hace de él tiene mucho que ver con ese desempeño social que altera e influye en gran medida en la vida de estas personas y en su desarrollo profesional.

Tendremos en el futuro que seguir investigando estos aspectos y en la medida de lo posible proporcionar programas y/o tratamientos que palien estas dificultades que llegan a convertirse en déficits y que afectan a la persona y a su desempeño docente.

Fomentar programas cognitivos que trabajen estos aprendizajes y la adquisición de otros nuevos es sólo el principio de una tarea que tiene que comenzar por conseguir una mejora de las capacidades de los alumnos de hoy, docentes mañana.

\section{BIBLIOGRAFÍA}

Andreu, L., Sanz, M. y Serrat, E. (2009). Una propuesta de renovación metodológica en el marco del Espacio Europeo de Enseñanza Superior: los pequeños grupos de investigación cooperativos. Revista Electrónica Interuniversitaria de Formación del Profesorado, 12, (3), 111-126.

Anguera, M. T. (2000). Observación de conducta interactiva en contextos naturales. Aplicaciones. Barcelona: Universitat Barcelona.

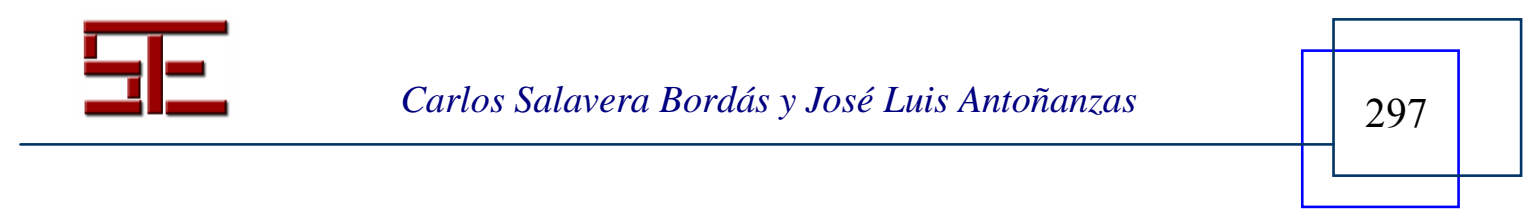




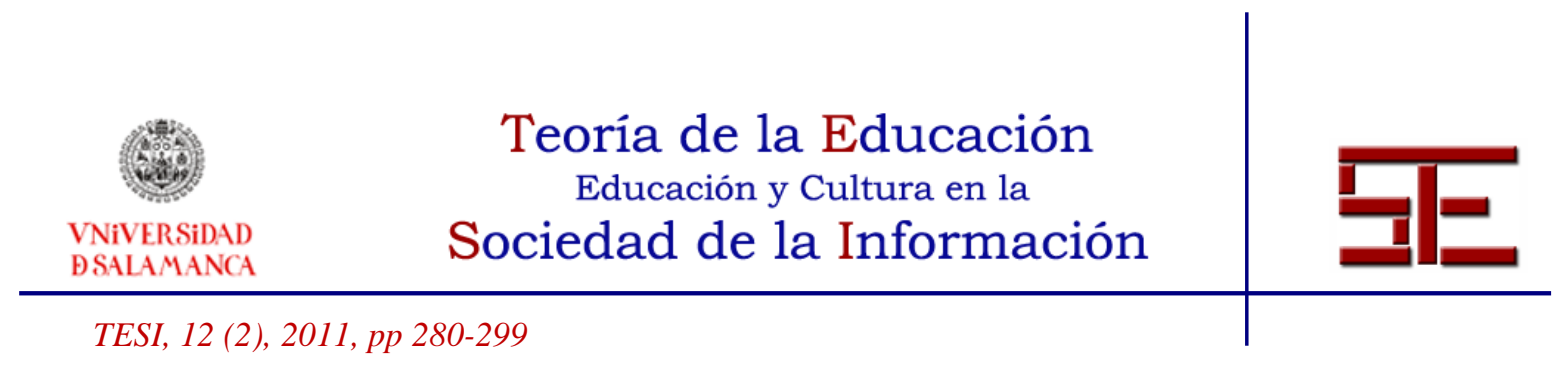

Caballo, V. (1993). Manual de evaluación y entrenamiento en habilidades sociales, Madrid: Siglo XXI.

Fernández, E. (2009). Aprendizaje experiencial, investigación-acción y creación organizacional de saber: la formación concebida como una zona de innovación profesional. Revista Electrónica Interuniversitaria de Formación del Profesorado, 12, (3), 39-57.

Gil, F. (1984). Entrenamiento en habilidades sociales. Granada:Alhambra Universidad. Goldstein, A.P., Sprafkin, R.P., Gershaw, N.J. y Klein, P. (1989). Habilidades sociales y autocontrol en la adolescencia, Barcelona: Martínez-Roca.

Gómez-López, J., Sanz, J. y Salavera, C. (2010). Las nuevas tecnologías de la informacion y comunicación en alumnos de secundaria del sistema educativo español: actitudes y tecnoestrés (en prensa).

Kelly, J. A. (1987). Entrenamiento de las habilidades sociales, Bilbao: Descleé de Brouwer.

Monereo, C. (1997). Las estrategias de aprendizaje. Barcelona: Edebé.

Muria, I. (1994). La enseñanza de las estrategias de aprendizaje y las habilidades metacognitivas. Perfiles Educativos, 65, 63-72.

Nerdrum, P. (1997). Maintenance of the effect of training in communication skills: a controlled follow-up study of level of communicated empathy. British Journal Social Worker, 27, 705-722.

Prichard, J.S., Stratford, R.J. y Bizo, L.A. (2006). Team-skills training enhances collaborative learning. Learning and Instruction, 16, (3), 256-265.

Ramos, F. y Manga, D. (1991). Alteraciones del lenguaje. En Belloch, A. Manual de Psicopatología. Madrid: McGraw-Hill.

Renzulli, J.S. (2010). El rol del profesor en el desarrollo del talento. Revista Electrónica Interuniversitaria de Formación del Profesorado, 13, (1), 33-40.

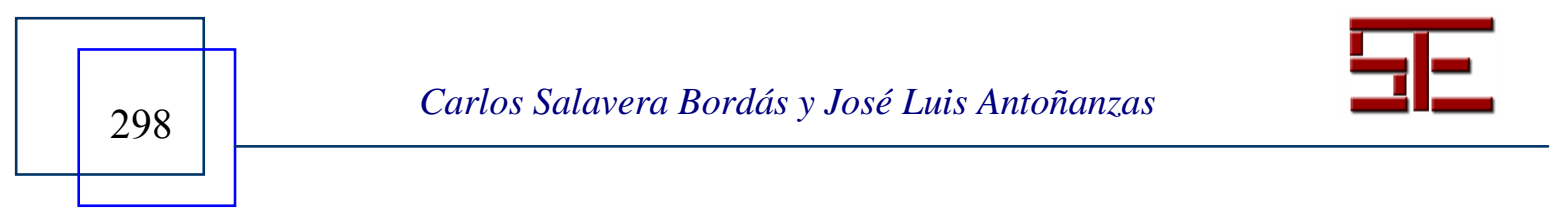




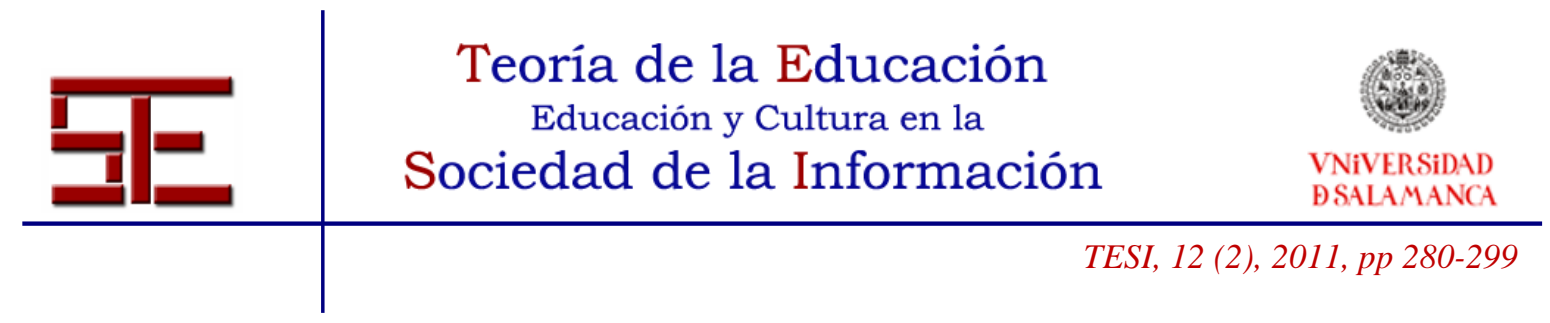

Salavera, C. (1998). Un programa de habilidades conversacionales con esquizofrénicos. Cuadernos de ICIJA, 4, 132-148.

- (1999). Análisis de patologías del lenguaje en la esquizofrenia: aspectos psicológicos. Cuadernos de ICIJA, 6, 185-192.

Salavera, C. y Puyuelo, M. (2010). Aspectos semánticos y pragmáticos en personas con esquizofrenia. Revista de Logopedia, Foniatría y Audiología, 30, 84-93.

Salavera, C., Tricás, J.M, Lucha, M.O., Gaspar, M.E., Herranz, F. y Jiménez, A. (1999). Licenciatura en fisioterapia: ¿solución a la ansiedad en los diplomados desempleados?. Anales de Ciencias de la Salud, 2, 127-138.

Soler, E., Núñez, J.C., González-Pienda, J.A. y Álvarez, L. (2002). Estrategias de aprendizaje: concepto, evaluación e intervención, Madrid: Pirámide.

Van Nuland, M., Thijs, G., Van Royen, P., Van den Noortgate, W. y Goedhuys, J. (2010). Vocational trainees' views and experiences regarding the learning and teaching of communication skills in general practice. Patient Education and Counseling, 78, (1), 65-71.

Para citar el presente artículo puede utilizar la siguiente referencia:

Salavera Bordás, C. y Antoñanzas, J. L. (2011). Las habilidades de comunicación como elemento de innovación educativa: estrategias de aprendizaje, en Barrios Vicente, I. M. (Coord.) Mujeres y la sociedad de la Información. Revista Teoría de la Educación: Educación y Cultura en la Sociedad de la Información. Vol. 12, $\mathrm{n}^{\circ}$ 2. Universidad de Salamanca, pp. 280-299 [Fecha de consulta: dd/mm/aaaa].

http://campus.usal.es/ revistas_trabajo/index.php/revistatesi/article/view/8282/8286

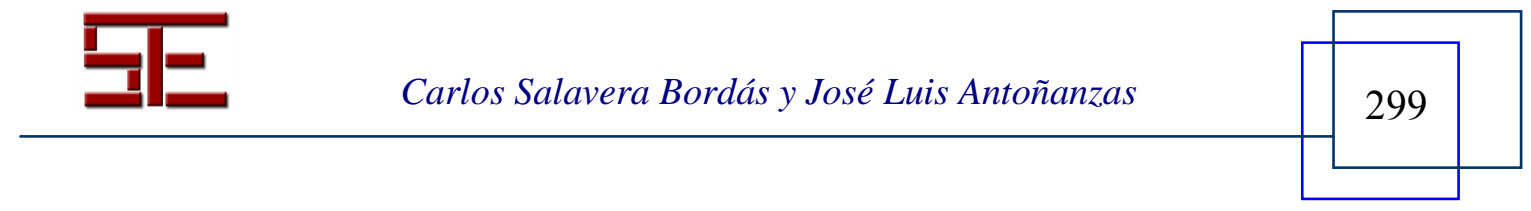

